\title{
Overview of the Finite Element Method
}

\author{
M. Kuczmann \\ Széchenyi István University, Department of Automation \\ Egyetem tér 1, H9026, Gyốr, Hungary \\ E-mail: kuczmann@sze.hu
}

Abstract: $\quad$ By using scalar and vector potentials, Maxwell's equations can be transformed into partial differential equations. Generally, the partial differential equations can be solved by numerical methods. One of these numerical methods is the finite element method, which is based on the weak formulation of the partial differential equations. The basis of numerical techniques is to reduce the partial differential equations to algebraic ones whose solution gives an approximation of the unknown potentials and electromagnetic field quantities. This reduction can be done by discretizing the partial differential equations in time if necessary and in space. The potential functions, the approximation method and the generated mesh distinguish the numerical field solvers. This paper summarize the finite element method as a CAD technique in electrical engineering to obtain the electromagnetic field quantities in the case of static magnetic field and eddy current field problems. Here, we show how to discretize the analyzed domain with finite elements, how to approximate potential functions with nodal and vector shape functions and how to build up the system of equations, which solution obtain the unknown potentials.

Keywords: Maxwell's equations, weak formulation, finite element method

\section{Introduction}

This paper is based on the book [1].

The Finite Element Method (FEM) is the most popular and the most flexible numerical technique to determine the approximate solution of the partial differential equations in engineering. For example, commercially available FEM software package is COMSOL Multiphysics, which is able to solve one, two and three-dimensional problems. A free mesh generator with a built-in CAD engine and post-processor is Gmsh.

The main steps of simulation with FEM are illustrated in Fig. 1. Firstly, in the model specification phase, the model of the real life problem, which simulation require electromagnetic field calculations must be set up, i.e. we have to find out the partial differential 


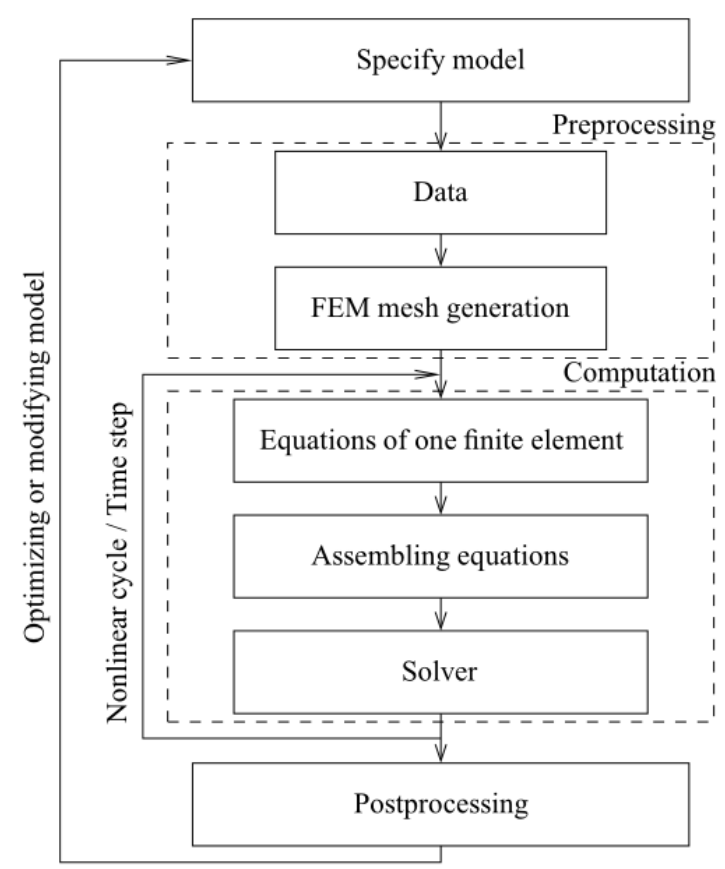

Figure 1. Steps of simulation by FEM.

equations, which must be solved with prescribed boundary and continuity conditions. We have to find out, whether it is a linear or a nonlinear problem and how the characteristics look like. After selecting potentials, the weak formulation of these partial differential equations must be worked out as well. It is depending on the problem, of course, but the chosen mathematical model of the arrangement should be adequate to calculate electromagnetic field quantities in the given accuracy. The geometry of the problem must be defined by a CAD software tool, e.g. by using a user friendly interface, see e.g. Fig. 2.

The next step is the preprocessing task. Here we have to give the values of different parameters, such as the material properties, i.e. the constitutive relations, the excitation signal and the others. The geometry can be simplified according to symmetries or axial symmetries.

The geometry of the problem must be discretized by a FEM mesh. The fundamental idea of FEM is to divide the problem region to be analyzed into smaller finite elements with given shape. A finite element can be e.g. triangle or quadrangle in 2D, e.g. tetrahedron or hexahedra in 3D. A triangle has three vertices 1, 2 and 3 numbered counter-clockwise and has 3 edges. The quadrangle element has 4 nodes and 4 edges. A tetrahedral element has 4 


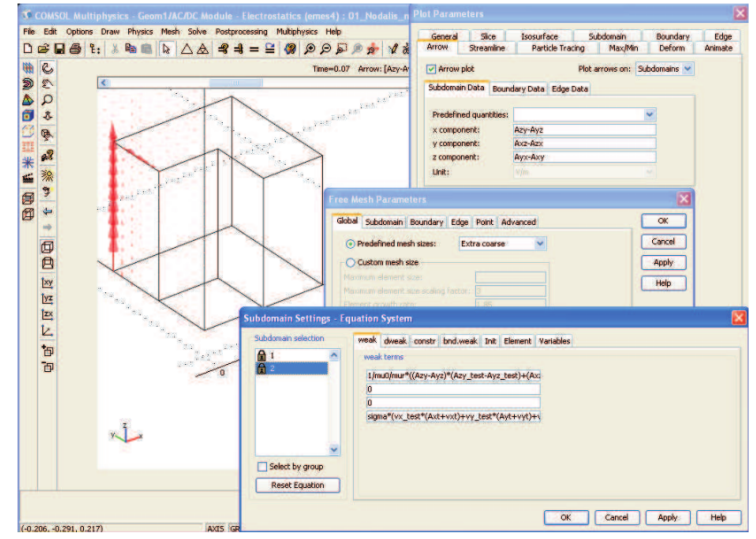

Figure 2. COMSOL Multiphysics, a CAD environment to solve electromagnetic field problems.

vertices and 6 edges and a hexahedral element has 8 nodes and 12 edges.

There are some simple rules, how to generate a mesh. Neither overlapping nor holes are allowed in the generated finite element mesh. If material interface are included in the problem region, the configuration of mesh must be adapted to these boundaries, i.e. interfaces coincide with finite element interfaces.

FEM mesh, as two illustrations, generated by COMSOL Multiphysics can be seen in Fig. 3 and in Fig. 4. The first 2D illustration (Fig. 3) shows the mesh of a horseshoeshaped permanent magnet. The two ends are pre-magnetized in different directions. The second illustration (Fig. 4) presents a model of a micro-scale square inductor, used for LC bandpass filters in microelectromechanical systems. The model geometry consists of the spiral-shaped inductor and the air surrounding it (the mesh in air is not shown). The outer dimensions of the model geometry are around $0.3 \mathrm{~mm}$. These illustrations are cited from the Model Documentation of COMSOL Multiphysics.

The next step in FEM simulations is solving the problem. The FEM equations, based on the weak formulations, must be set up in the level of one finite element, then these equations must be assembled through the FEM mesh. Assembling means that the global system of equations is built up, which solution is the approximation of the introduced potential. The obtained global system of algebraic equations is linear, or nonlinear but linearized, depending on the medium to be analyzed. Then this global system of equations must be solved by a solver. The computation may contain iteration if the constitutive equations are nonlinear. This is the situation when simulating ferromagnetic materials with nonlinear characteristics. Iteration means that the system of equations must be set up and 


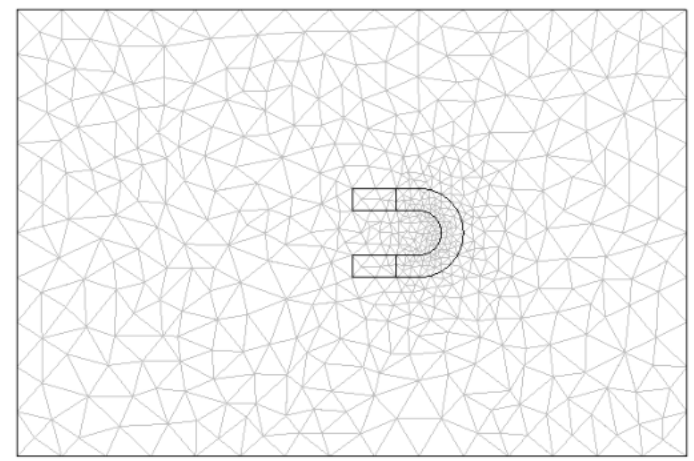

Figure 3. COMSOL model of a permanent magnet, geometry is meshed by triangles.

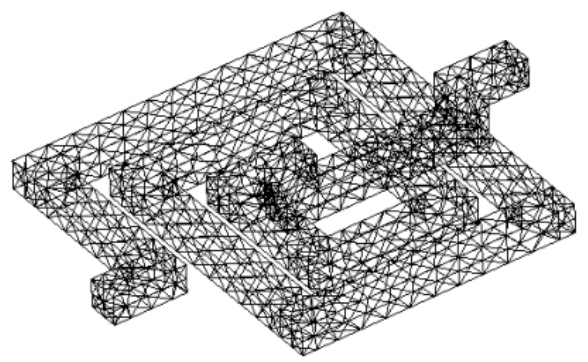

Figure 4. COMSOL model of a micro-scale square inductor, geometry is discretized by tetrahedral shape finite elements.

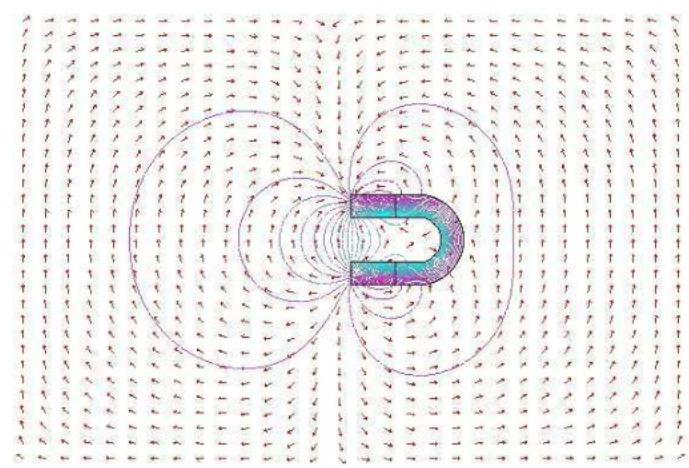

Figure 5. COMSOL solution of the static magnetic field generated by a permanent magnet. 


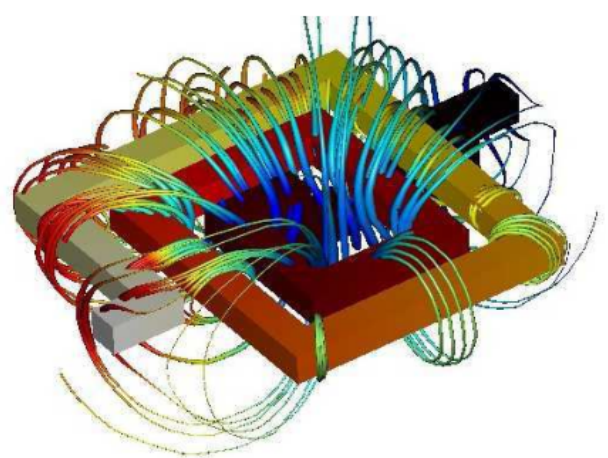

Figure 6. Electric potential in the device and magnetic flux lines around the device, the problem has been solved by COMSOL.

must be solved step by step until convergence is reached. If the problem is time dependent, then the solution must be worked out at every discrete time instant.

The result of computations is the approximated potential value in the FEM mesh. Any electromagnetic field quantity (e.g. magnetic field intensity, or magnetic flux density, etc.) can be calculated by using the potentials at the postprocessing stage. Capacitance, inductance, energy, force and other quantities can also be calculated. The postprocessing give a chance to modify the geometry, the material parameters or the FEM mesh to get more accurate result. The COMSOL Multiphysics has been used to show two examples about postprocessing. The pattern of the magnetic field around the permanent magnet is well known through experiments (see Fig. 5). Figure 6 shows the electric potential in the inductor and the magnetic flux lines. The thickness of the flow lines represents the magnitude of the magnetic flux.

\section{Approximating potentials with shape functions}

The potential function can be scalar valued (e.g. the magnetic scalar potential $\Phi$, or the electric scalar potential $V$ ), or vector valued (e.g. the current vector potential $\boldsymbol{T}$, or the magnetic vector potential $\boldsymbol{A}$ ).

The scalar potential functions can be approximated by so-called nodal shape functions and the vector potential functions can be approximated by either nodal or so-called vector shape functions, also called edge shape functions. Generally, a shape function is a simple continuous polynomial function defined in a finite element and it is depending on the type of the used finite element.

Shape functions have the following general properties: 
(i) Each shape function is defined in the entire problem region;

(ii) Each scalar shape function corresponds to just one nodal point and each vector shape function corresponds to just one edge;

(iii) Each scalar shape function is nonzero over just those finite elements that contain its nodal point and equals to zero over all other elements. Each vector shape function in nonzero over just those finite elements that contain its edge and equals to zero over all other elements;

(iv) The scalar shape function has a value unity at its nodal point and zero at all other nodal points. The line integral of a vector shape function is equal to one along its edge and the line integral of it is equal to zero along the other edges;

(v) The shape functions are linearly independent, i.e. no shape function equals a linear combination of the other shape functions.

The accuracy of solution obtained by FEM can be increased in three ways. The first one is increasing the number of finite elements, i.e. decreasing the element size. It is called $h$-FEM. The second way is to increase the degree of polynomials building up a shape function (e.g. using Lagrange or Legendre interpolation functions). This is the so-called $p$-FEM. The mixture of these methods results in $h p$-FEM. Potentials approximated by $h$-version or $p$-version are assigned in the indices of the potentials.

\subsection{Nodal finite elements}

Scalar potential functions can be represented by a linear combination of shape functions associated with nodes of the finite element mesh. Within a finite element, a scalar potential function $\Phi=\Phi(\boldsymbol{r}, t)$ is approximated by

$$
\Phi \simeq \sum_{i=1}^{m} N_{i} \Phi_{i},
$$

where $N_{i}=N_{i}(\boldsymbol{r})$ and $\Phi_{i}=\Phi_{i}(t)$ are the nodal shape functions and the value of potential function corresponding to the $i^{\text {th }}$ node, respectively. The number of degrees of freedom is $m=2$ in $1 \mathrm{D}$ problems, $m=3$ in a $2 \mathrm{D}$ problem using triangular FEM mesh and $m=4$ in a $3 \mathrm{D}$ arrangement meshed by tetrahedral elements and the shape functions are linear. The nodal shape functions can be defined by the relation

$$
N_{i}= \begin{cases}1, & \text { at the node } i, \\ 0, & \text { at other nodes }\end{cases}
$$

(i) In 1D, the linear shape functions can be build up by

$$
N_{1}=\frac{x_{2}-x}{x_{2}-x_{1}}, \text { and } \quad N_{2}=\frac{x-x_{1}}{x_{2}-x_{1}},
$$


where $x_{1}$ and $x_{2}$ are the coordinates of the boundaries of one finite element. The linear shape functions are plotted in Fig. 7. It is easy to control the equation (2).

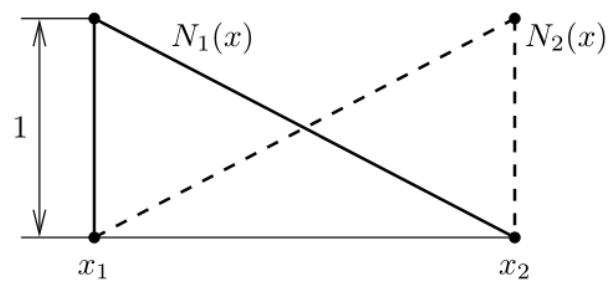

Figure 7. The $1 D$ linear shape functions $N_{1}(x)$ and $N_{2}(x)$.

If the values of the potential are known in the two boundary points $x_{1}$ and $x_{2}$, then the potential can be determined easily inside the finite element $x_{1} \leq x \leq x_{2}$ as (see Fig. 8)

$$
\Phi=N_{1} \Phi_{1}+N_{2} \Phi_{2}=\frac{x_{2}-x}{x_{2}-x_{1}} \Phi_{1}+\frac{x-x_{1}}{x_{2}-x_{1}} \Phi_{2}
$$

Of course, it is valid in the other finite elements as well, e.g. if $x_{2} \leq x \leq x_{3}$, then

$$
\Phi=N_{1} \Phi_{2}+N_{2} \Phi_{3}=\frac{x_{3}-x}{x_{3}-x_{2}} \Phi_{2}+\frac{x-x_{2}}{x_{3}-x_{2}} \Phi_{3},
$$

and $N_{1}, N_{2}$ are shifted to the second finite element.

The scalar potential is continuous in the whole $1 \mathrm{D}$ region. It is noted here that the accuracy of approximation can be increased by decreasing the length of the elements,

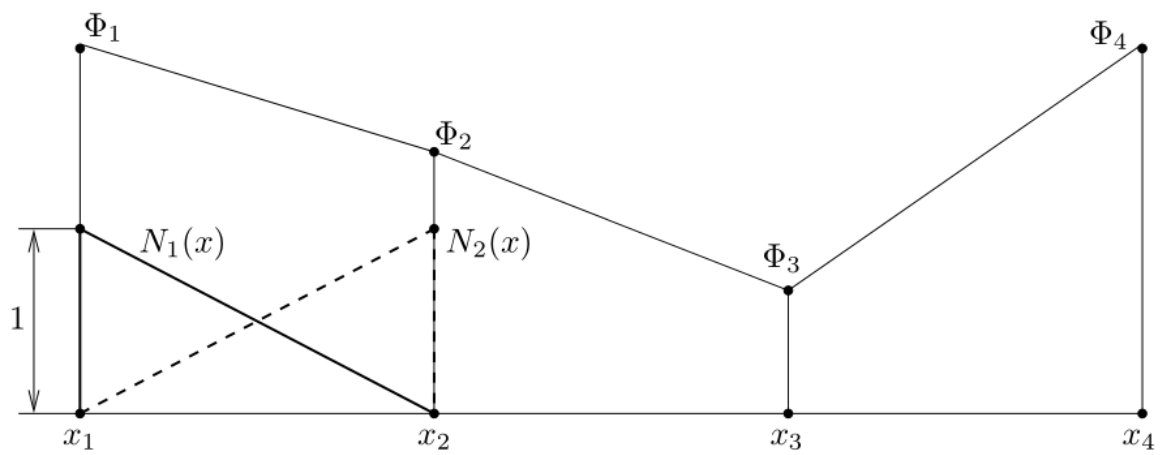

Figure 8. Known potential values are approximated by linear functions. 
especially where the rate of change of the solution is large, e.g. between $x_{3}$ and $x_{4}$ in Fig. 8. Here, the mesh can be very fine and higher order approximation can results in better solution.

One way to build up higher order shape functions is using Lagrange interpolation functions, defined by the formula

$$
N_{i}(x)=\prod_{j=1, j \neq i}^{m} \frac{x-x_{j}}{x_{i}-x_{j}} .
$$

The order is $m-1$ and $N_{i}(x)$ is equal to one in the node $i$ and equal to zero in all the other nodes. Here, second and third order approximations are shown.

The second order approximation can be defined by 3 quadratic shape functions (i.e. $m=3$ in (1), see Fig. 9),

$$
\begin{aligned}
& N_{1}=\frac{\left(x-x_{2}\right)\left(x-x_{3}\right)}{\left(x_{1}-x_{2}\right)\left(x_{1}-x_{3}\right)}, \\
& N_{2}=\frac{\left(x-x_{1}\right)\left(x-x_{3}\right)}{\left(x_{2}-x_{1}\right)\left(x_{2}-x_{3}\right)}, \\
& N_{3}=\frac{\left(x-x_{1}\right)\left(x-x_{2}\right)}{\left(x_{3}-x_{1}\right)\left(x_{3}-x_{2}\right)},
\end{aligned}
$$

and the new point $x_{3}$ is placed in the center of the element,

$$
x_{3}=\frac{x_{1}+x_{2}}{2} .
$$

The third order approximation can be defined by 4 cubic shape functions ( $m=4$ in (1), see Fig. 10),

$$
N_{1}=\frac{\left(x-x_{2}\right)\left(x-x_{3}\right)\left(x-x_{4}\right)}{\left(x_{1}-x_{2}\right)\left(x_{1}-x_{3}\right)\left(x_{1}-x_{4}\right)},
$$

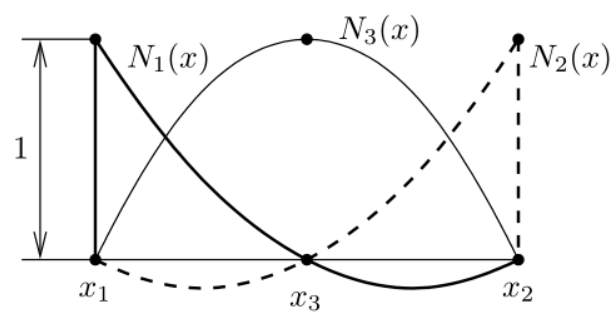

Figure 9. The 1D quadratic shape functions $N_{1}(x), N_{2}(x)$ and $N_{3}(x)$. 


$$
\begin{aligned}
& N_{2}=\frac{\left(x-x_{1}\right)\left(x-x_{3}\right)\left(x-x_{4}\right)}{\left(x_{2}-x_{1}\right)\left(x_{2}-x_{3}\right)\left(x_{2}-x_{4}\right)}, \\
& N_{3}=\frac{\left(x-x_{1}\right)\left(x-x_{2}\right)\left(x-x_{4}\right)}{\left(x_{3}-x_{1}\right)\left(x_{3}-x_{2}\right)\left(x_{3}-x_{4}\right)}, \\
& N_{4}=\frac{\left(x-x_{1}\right)\left(x-x_{2}\right)\left(x-x_{3}\right)}{\left(x_{4}-x_{1}\right)\left(x_{4}-x_{2}\right)\left(x_{4}-x_{3}\right)},
\end{aligned}
$$

and the new points $x_{3}$ and $x_{4}$ are placed inside the element as

$$
x_{3}=\frac{1\left(x_{1}+x_{2}\right)}{3}, \quad x_{4}=\frac{2\left(x_{1}+x_{2}\right)}{3} .
$$

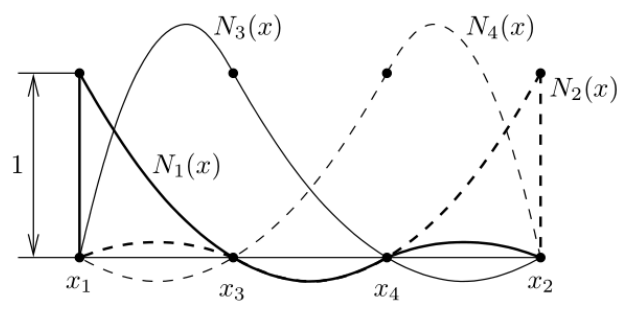

Figure 10. The 1D cubic shape functions $N_{1}(x), N_{2}(x), N_{3}(x)$ and $N_{4}(x)$.

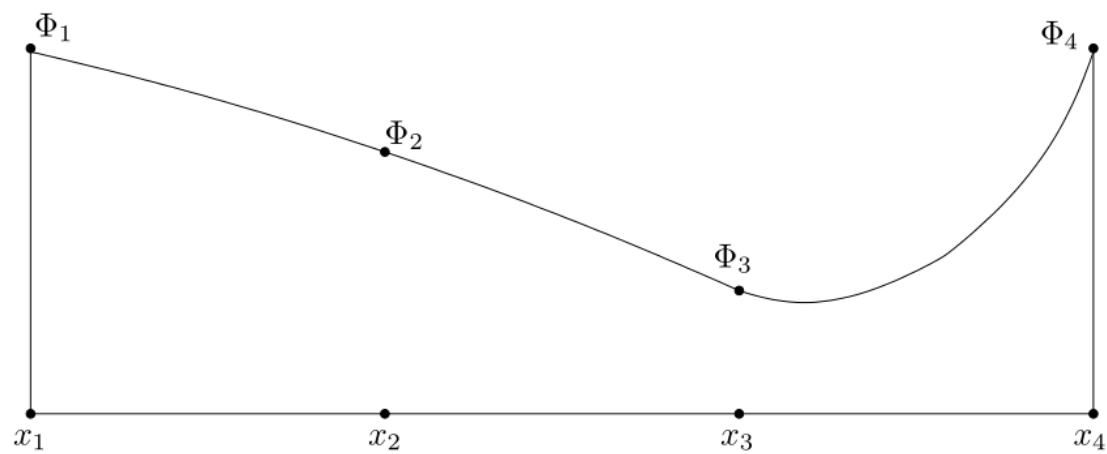

Figure 11. Known potential values are approximated by quadratic functions.

With this technique, the interpolation functions of any order can be defined and the equation (2) can be controlled.

Figure 11 shows the higher order approximation of the potential plotted in Fig. 8. This illustration shows the applicability of higher order functions. 
(ii) 2D linear shape functions can be built up as follows when using a finite element mesh with triangular finite elements. Linear basis functions can be introduced by using the so-called barycentric coordinate system in a triangle as follows. The area of a triangle is denoted by $\Delta$ and it can be calculated as

$$
\Delta=\frac{1}{2}\left|\begin{array}{lll}
1 & x_{1} & y_{1} \\
1 & x_{2} & y_{2} \\
1 & x_{3} & y_{3}
\end{array}\right|
$$

where $\left(x_{1}, y_{1}\right),\left(x_{2}, y_{2}\right)$ and $\left(x_{3}, y_{3}\right)$ are the coordinates of the three nodes of the triangle in the global coordinate system building an anticlockwise sequence. The area functions (see Fig. 12) of a given point inside the triangle with coordinates $(x, y)$ can be calculated as

$$
\Delta_{1}=\frac{1}{2}\left|\begin{array}{ccc}
1 & x & y \\
1 & x_{2} & y_{2} \\
1 & x_{3} & y_{3}
\end{array}\right|, \Delta_{2}=\frac{1}{2}\left|\begin{array}{ccc}
1 & x_{1} & y_{1} \\
1 & x & y \\
1 & x_{3} & y_{3}
\end{array}\right|, \Delta_{3}=\frac{1}{2}\left|\begin{array}{ccc}
1 & x_{1} & y_{1} \\
1 & x_{2} & y_{2} \\
1 & x & y
\end{array}\right|,
$$

i.e. $\Delta_{1}=\Delta_{1}(x, y), \Delta_{2}=\Delta_{2}(x, y)$ and $\Delta_{3}=\Delta_{3}(x, y)$ are depending on the coordinates $x$ and $y$.

The barycentric coordinates $L_{i}=L_{i}(x, y)$ can be defined by the above area functions as

$$
L_{i}=\frac{\Delta_{i}}{\Delta}, \quad i=1,2,3
$$

Three linear shape functions $N_{i}=N_{i}(x, y)$ can be described as

$$
N_{i}=L_{i}, \quad i=1,2,3 .
$$

The shape function $N_{i}$ is equal to 1 at the $i^{\text {th }}$ node of the triangle and it is equal to zero at the other two nodes, because $\Delta_{i}$ is equal to $\Delta$ at node $i$ and it is equal to zero at the other two nodes. That is why the relation (2) is satisfied. It is obvious that the three shape functions are linearly independent.

The linear shape functions $N_{i}(i=1,2,3)$ vary linearly over the triangle, because the fraction $\Delta_{i} / \Delta$ measures the perpendicular distance of the point $(x, y)$ toward the vertex opposite to node $i$ as it is illustrated in Fig. 13 and the linear shape function is constant along such a line. The three linear shape functions are shown in Fig. 14.

If the potential at the nodes is known, then a linear approximation of the potential function can be represented by (1). The derivative of a first order approximation is zeroth order, i.e. constant. The magnetic field intensity $\boldsymbol{H}$, or the magnetic flux density $\boldsymbol{B}$ are constant within a triangle, if these are obtained from a first order approximation by $\boldsymbol{H}=-\nabla \Phi$, or $\boldsymbol{B}=\nabla \times \boldsymbol{A}$. This may results in inaccurate solution. This is the reason why higher order approximations are studied. Here, only the second and the third order approximations are shown. 


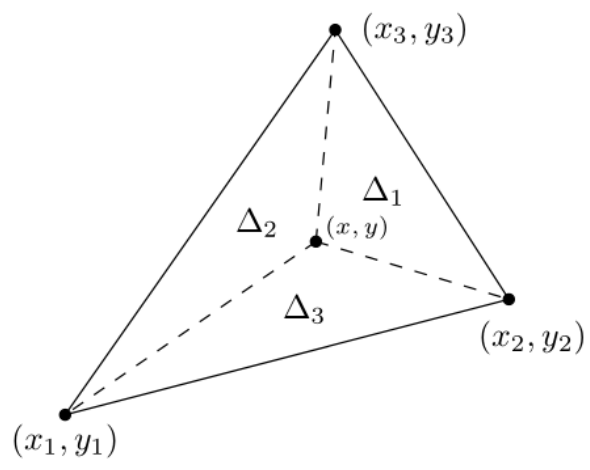

Figure 12. The area function of a triangle.

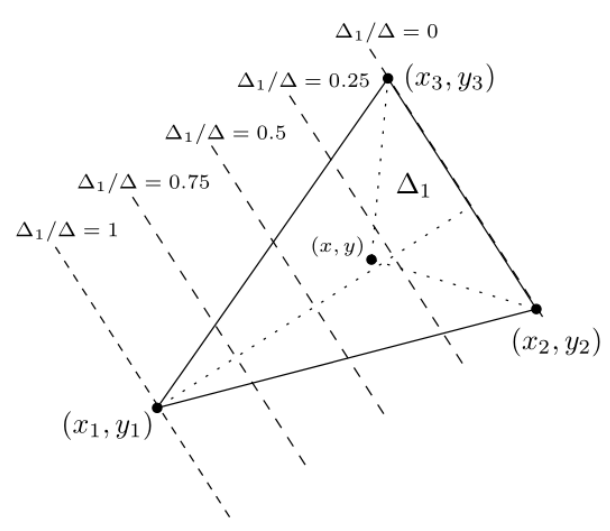

Figure 13. Fraction $\Delta_{i} / \Delta$ measures the perpendicular distance of the point $(x, y)$ toward the vertex opposite to node $i$ (here $i=1$ ).

Higher order shape functions can also be built up by using the barycentric coordinates $L_{1}, L_{2}$ and $L_{3}$ introduced above in (18). 


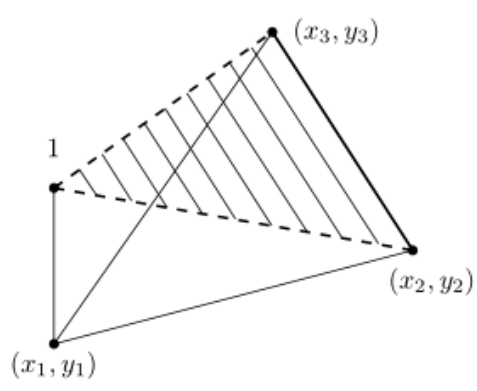

(a) $N_{1}(x, y)$

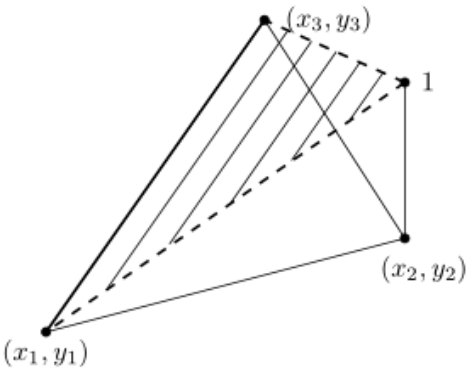

(b) $N_{2}(x, y)$

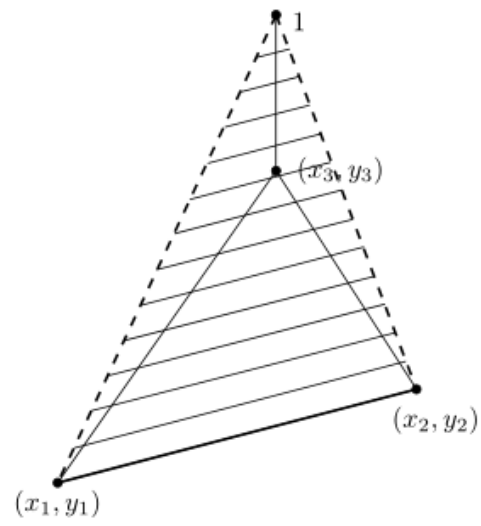

(c) $N_{3}(x, y)$

Figure 14. The 2D linear shape functions $N_{1}(x, y), N_{2}(x, y)$ and $N_{3}(x, y)$.

A polynomial of order $n$ must contain all possible terms $x^{p} y^{q}, 0 \leq p+q \leq n$, as it is presented by Pascal's triangle,

$$
\begin{aligned}
& 1 \\
& x \quad y \\
& x^{2} \quad x y \quad y^{2} \\
& \begin{array}{lllll}
x^{3} & x^{2} y & x y^{2} & y^{3} & \ldots
\end{array}
\end{aligned}
$$

The first row contains the only one term of the zeroth order polynomials, the second, third and fourth rows contain the terms of the first, second and third order polynomials. Pascal's triangle can be used to generate the elements of a polynomial with given order. Such a polynomial contains

$$
m=\frac{(n+1)(n+2)}{2}
$$


elements altogether, i.e. $m=1, m=3, m=6$ and $m=10$ in the case of zeroth, first, second and third order polynomials. It means that $m$ coefficients must be expressed, finally $m$ points must be placed within a triangle. Pascal's triangle can be continued, of course.

The interpolation function of order $n$ can be constructed as

$$
N_{i}=P_{I}^{n}\left(L_{1}\right) P_{J}^{n}\left(L_{2}\right) P_{K}^{n}\left(L_{3}\right), \quad \text { where } I+J+K=n,
$$

and the integers $I, J$ and $K$ label the nodes within the triangle, resulting in a numbering scheme. Figure 15, Fig. 16 and Fig. 17 illustrate the numbering scheme of the first, the second and the third order approximations. It is noted that points must be inserted not only the edges, but inside the triangle, if $n>2$.

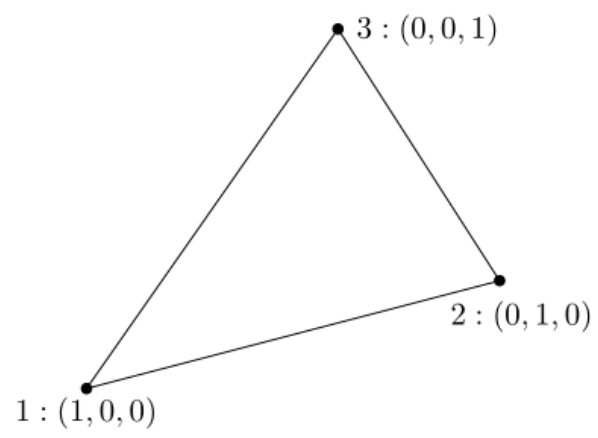

Figure 15. Numbering scheme for linear element, $n=1$.

The polynomials $P_{I}^{n}\left(L_{1}\right), P_{J}^{n}\left(L_{2}\right)$ and $P_{K}^{n}\left(L_{3}\right)$ are defined as

$$
\begin{gathered}
P_{I}^{n}\left(L_{1}\right)=\prod_{p=0}^{I-1} \frac{n L_{1}-p}{I-p}=\frac{1}{I !} \prod_{p=0}^{I-1}\left(n L_{1}-p\right), \quad \text { if } \quad I>0, \\
P_{J}^{n}\left(L_{2}\right)=\prod_{p=0}^{J-1} \frac{n L_{2}-p}{J-p}=\frac{1}{J !} \prod_{p=0}^{J-1}\left(n L_{2}-p\right), \quad \text { if } \quad J>0, \\
P_{K}^{n}\left(L_{3}\right)=\prod_{p=0}^{K-1} \frac{n L_{3}-p}{K-p}=\frac{1}{K !} \prod_{p=0}^{K-1}\left(n L_{3}-p\right), \quad \text { if } \quad K>0,
\end{gathered}
$$

and as a definition

$$
P_{0}^{n}=1
$$


If $n=1$, then $m=3$, i.e. (see Fig. 15)

$$
\begin{aligned}
& N_{1}=P_{1}^{1}\left(L_{1}\right) P_{0}^{1}\left(L_{2}\right) P_{0}^{1}\left(L_{3}\right)=L_{1}, \\
& N_{2}=P_{0}^{1}\left(L_{1}\right) P_{1}^{1}\left(L_{2}\right) P_{0}^{1}\left(L_{3}\right)=L_{2}, \\
& N_{3}=P_{0}^{1}\left(L_{1}\right) P_{0}^{1}\left(L_{2}\right) P_{1}^{1}\left(L_{3}\right)=L_{3},
\end{aligned}
$$

since

$$
P_{1}^{1}\left(L_{i}\right)=\prod_{p=0}^{1-1} \frac{1 L_{i}-p}{1-p}=L_{i},
$$

as it was mentioned in (19).

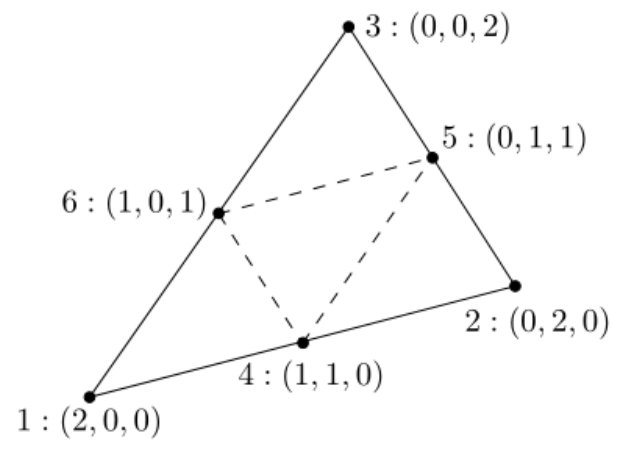

Figure 16. Numbering scheme for quadratic element, $n=2$.

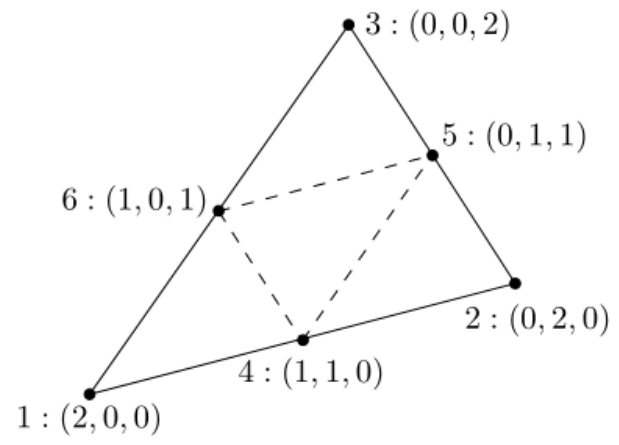

Figure 17. Numbering scheme for cubic element, $n=3$. 
If $n=2$, then $m=6$, i.e. (see Fig. 16)

$$
\begin{gathered}
N_{1}=P_{2}^{2}\left(L_{1}\right) P_{0}^{2}\left(L_{2}\right) P_{0}^{2}\left(L_{3}\right)=L_{1}\left(2 L_{1}-1\right), \\
N_{2}=P_{0}^{2}\left(L_{1}\right) P_{2}^{2}\left(L_{2}\right) P_{0}^{2}\left(L_{3}\right)=L_{2}\left(2 L_{2}-1\right), \\
N_{3}=P_{0}^{2}\left(L_{1}\right) P_{0}^{2}\left(L_{2}\right) P_{2}^{2}\left(L_{3}\right)=L_{3}\left(2 L_{3}-1\right), \\
N_{4}=P_{1}^{2}\left(L_{1}\right) P_{1}^{2}\left(L_{2}\right) P_{0}^{2}\left(L_{3}\right)=4 L_{1} L_{2}, \\
N_{5}=P_{0}^{2}\left(L_{1}\right) P_{1}^{2}\left(L_{2}\right) P_{1}^{2}\left(L_{3}\right)=4 L_{2} L_{3}, \\
N_{6}=P_{1}^{2}\left(L_{1}\right) P_{0}^{2}\left(L_{2}\right) P_{1}^{2}\left(L_{3}\right)=4 L_{1} L_{3},
\end{gathered}
$$

because

$$
P_{1}^{2}\left(L_{i}\right)=\prod_{p=0}^{1-1} \frac{2 L_{i}-p}{1-p}=2 L_{i}
$$

and

$$
P_{2}^{2}\left(L_{i}\right)=\prod_{p=0}^{2-1} \frac{2 L_{i}-p}{2-p}=\frac{2 L_{i}}{2} \frac{2 L_{i}-1}{1}=L_{i}\left(2 L_{i}-1\right) .
$$

Figure 18 shows the shape functions $N_{1}$ and $N_{4}$. The other shape functions look like these, $N_{2}$ and $N_{3}$ are the same as $N_{1}$, moreover $N_{5}$ and $N_{6}$ are the same as $N_{4}$, but they must be rotated to the corresponding nodes.

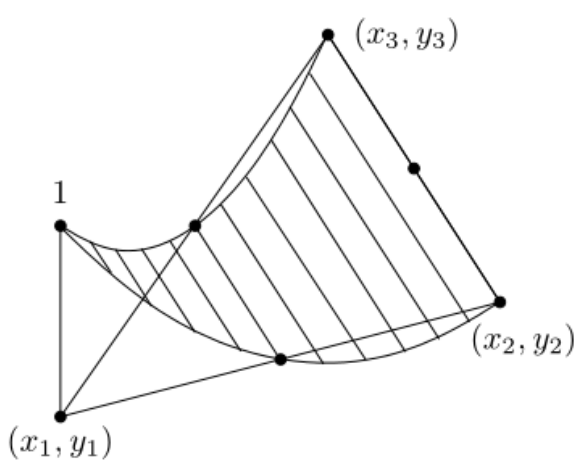

(a) $N_{1}(x, y)$

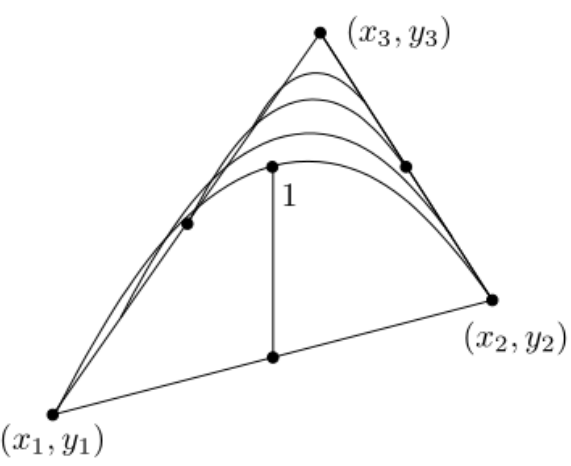

(b) $N_{4}(x, y)$

Figure 18. The 2D quadratic shape functions $N_{1}(x, y)$ and $N_{4}(x, y)$. 
Finally, if $n=3, m=10$, shape functions can be constructed as (see Fig. 19)

$$
\begin{gathered}
N_{1}=P_{3}^{3}\left(L_{1}\right) P_{0}^{3}\left(L_{2}\right) P_{0}^{3}\left(L_{3}\right)=\frac{1}{2} L_{1}\left(3 L_{1}-1\right)\left(3 L_{1}-2\right), \\
N_{2}=P_{0}^{3}\left(L_{1}\right) P_{3}^{3}\left(L_{2}\right) P_{0}^{3}\left(L_{3}\right)=\frac{1}{2} L_{2}\left(3 L_{2}-1\right)\left(3 L_{2}-2\right), \\
N_{3}=P_{0}^{3}\left(L_{1}\right) P_{0}^{3}\left(L_{2}\right) P_{3}^{3}\left(L_{3}\right)=\frac{1}{2} L_{3}\left(3 L_{3}-1\right)\left(3 L_{3}-2\right), \\
N_{4}=P_{2}^{3}\left(L_{1}\right) P_{1}^{3}\left(L_{2}\right) P_{0}^{3}\left(L_{3}\right)=\frac{9}{2} L_{1}\left(3 L_{1}-1\right) L_{2}, \\
N_{5}=P_{1}^{3}\left(L_{1}\right) P_{2}^{3}\left(L_{2}\right) P_{0}^{3}\left(L_{3}\right)=\frac{9}{2} L_{2}\left(3 L_{2}-1\right) L_{1}, \\
N_{6}=P_{0}^{3}\left(L_{1}\right) P_{2}^{3}\left(L_{2}\right) P_{1}^{3}\left(L_{3}\right)=\frac{9}{2} L_{2}\left(3 L_{2}-1\right) L_{3}, \\
N_{7}=P_{0}^{3}\left(L_{1}\right) P_{1}^{3}\left(L_{2}\right) P_{2}^{3}\left(L_{3}\right)=\frac{9}{2} L_{3}\left(3 L_{3}-1\right) L_{2}, \\
N_{8}=P_{1}^{3}\left(L_{1}\right) P_{0}^{3}\left(L_{2}\right) P_{2}^{3}\left(L_{3}\right)=\frac{9}{2} L_{3}\left(3 L_{3}-1\right) L_{1}, \\
N_{9}=P_{2}^{3}\left(L_{1}\right) P_{0}^{3}\left(L_{2}\right) P_{1}^{3}\left(L_{3}\right)=\frac{9}{2} L_{1}\left(3 L_{1}-1\right) L_{3}, \\
N_{10}=P_{1}^{3}\left(L_{1}\right) P_{1}^{3}\left(L_{2}\right) P_{1}^{3}\left(L_{3}\right)=27 L_{1} L_{2} L_{3},
\end{gathered}
$$

because

$$
P_{1}^{3}\left(L_{i}\right)=\prod_{p=0}^{1-1} \frac{3 L_{i}-p}{1-p}=3 L_{i}
$$

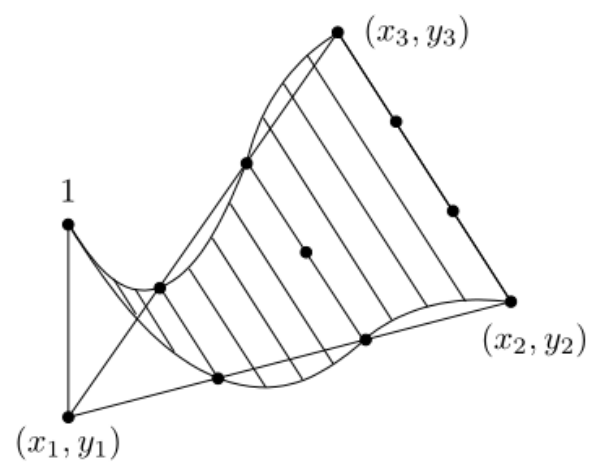

(a) $N_{1}(x, y)$

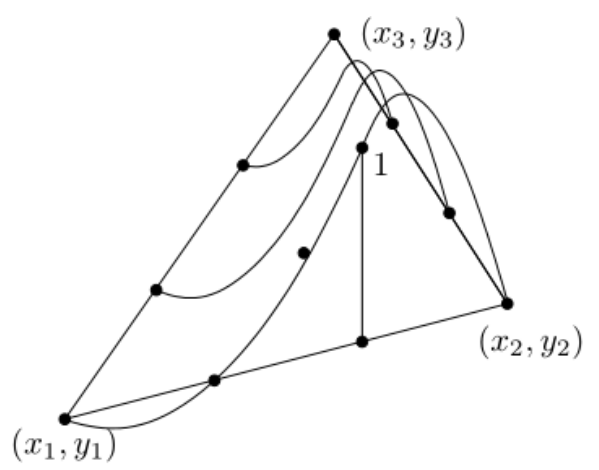

(b) $N_{5}(x, y)$

Figure 19. The $2 D$ cubic shape functions $N_{1}(x, y)$ and $N_{5}(x, y)$. 


$$
\begin{gathered}
P_{2}^{3}\left(L_{i}\right)=\prod_{p=0}^{2-1} \frac{3 L_{i}-p}{2-p}=\frac{3 L_{i}}{2} \frac{3 L_{i}-1}{1}=\frac{3}{2} L_{i}\left(3 L_{i}-1\right), \\
P_{3}^{3}\left(L_{i}\right)=\prod_{p=0}^{3-1} \frac{3 L_{i}-p}{3-p}=\frac{3 L_{i}}{3} \frac{3 L_{i}-1}{2} \frac{3 L_{i}-2}{1} \\
=\frac{1}{2} L_{i}\left(3 L_{i}-1\right)\left(3 L_{i}-2\right) .
\end{gathered}
$$

These functions satisfy the condition (2). Figure 19 shows the shape functions $N_{1}$ and $N_{5}$, as examples. The other shape functions look like these, $N_{2}$ and $N_{3}$ are the same as $N_{1}, N_{4}, N_{6}, N_{7}, N_{8}$ and $N_{9}$ look like $N_{5}$, but they must be imagined at the corresponding nodes. The shape function $N_{10}$ is equal to one at the center of mass of the triangle and equal to zero on the other nine nodes.

The scalar potential along any edge of a triangle is the linear combination of the values defined in the points of this edge (see Fig. 15, Fig. 16, Fig. 17), so that if two triangles share the same vertice, the potential will be continuous across the interface element boundary. This means that the approximate solution is continuous everywhere, however, its normal derivate is not.

It is easy to see that the $1 \mathrm{D}$ shape functions are the same as the functions along the edges of a triangle.

(iii) 3D linear shape functions can be worked out as follows when using tetrahedral finite elements. Linear basis functions can be introduced again by using the barycentric coordinate system. The volume of a tetrahedron is denoted by $V$ and it can be expressed as

$$
V=\frac{1}{6}\left|\begin{array}{lll}
x_{4}-x_{1} & y_{4}-y_{1} & z_{4}-z_{1} \\
x_{4}-x_{2} & y_{4}-y_{2} & z_{4}-z_{2} \\
x_{4}-x_{3} & y_{4}-y_{3} & z_{4}-z_{3}
\end{array}\right|,
$$

where $\left(x_{1}, y_{1}, z_{1}\right),\left(x_{2}, y_{2}, z_{2}\right),\left(x_{3}, y_{3}, z_{3}\right)$ and $\left(x_{4}, y_{4}, z_{4}\right)$ are the coordinates of the four nodes of the tetrahedron as shown in Fig. 20. The volume functions according to a given point inside the tetrahedron with coordinates $(x, y, z)$ can be calculated as

$$
\begin{gathered}
V_{1}=\frac{1}{6}\left|\begin{array}{ccc}
x_{4}-x & y_{4}-y & z_{4}-z \\
x_{4}-x_{2} & y_{4}-y_{2} & z_{4}-z_{2} \\
x_{4}-x_{3} & y_{4}-y_{3} & z_{4}-z_{3}
\end{array}\right|, \\
V_{2}=\frac{1}{6}\left|\begin{array}{ccc}
x_{4}-x_{1} & y_{4}-y_{1} & z_{4}-z_{1} \\
x_{4}-x & y_{4}-y & z_{4}-z \\
x_{4}-x_{3} & y_{4}-y_{3} & z_{4}-z_{3}
\end{array}\right|,
\end{gathered}
$$




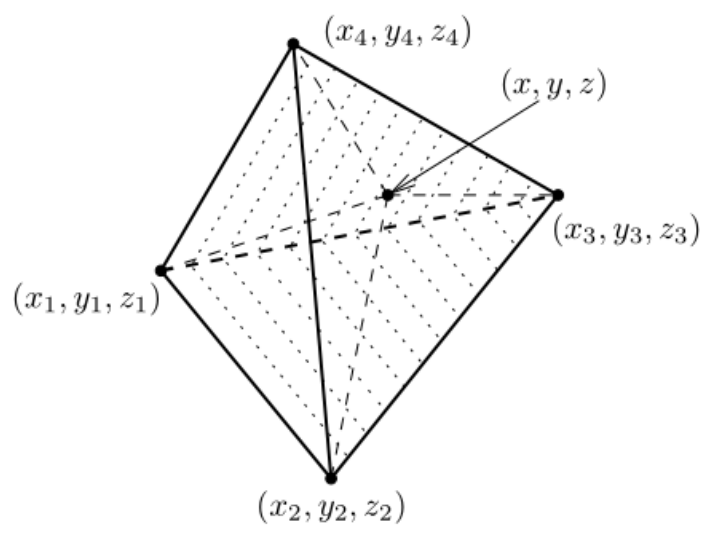

Figure 20. The volume functions in a tetrahedron.

$$
\begin{gathered}
V_{3}=\frac{1}{6}\left|\begin{array}{ccc}
x_{4}-x_{1} & y_{4}-y_{1} & z_{4}-z_{1} \\
x_{4}-x_{2} & y_{4}-y_{2} & z_{4}-z_{2} \\
x_{4}-x & y_{4}-y & z_{4}-z
\end{array}\right|, \\
V_{4}=\frac{1}{6}\left|\begin{array}{ccc}
x-x_{1} & y-y_{1} & z-z_{1} \\
x-x_{2} & y-y_{2} & z-z_{2} \\
x-x_{3} & y-y_{3} & z-z_{3}
\end{array}\right| .
\end{gathered}
$$

The barycentric coordinates $L_{i}=L_{i}(x, y, z)$ of a tetrahedron can be formulated as

$$
L_{i}=\frac{V_{i}}{V}, \quad i=1,2,3,4 .
$$

Four linear shape functions $N_{i}=N_{i}(x, y, z)$ correspondingly to the four nodes are

$$
N_{i}=L_{i}, \quad i=1,2,3,4 .
$$

A shape function $N_{i}$ is equal to 1 at the $i^{\text {th }}$ node of the tetrahedron, moreover it is equal to zero at the other three nodes and it is varying linearly within the tetrahedron, because the fraction $V_{i} / V$ measures the perpendicular distance of the point $(x, y, z)$ toward the facet opposite to node $i$ as it is illustrated in Fig. 21 and the linear shape function is constant along such a surface. That is why the relation (2) is satisfied. It is obvious that the four shape functions are linearly independent. 


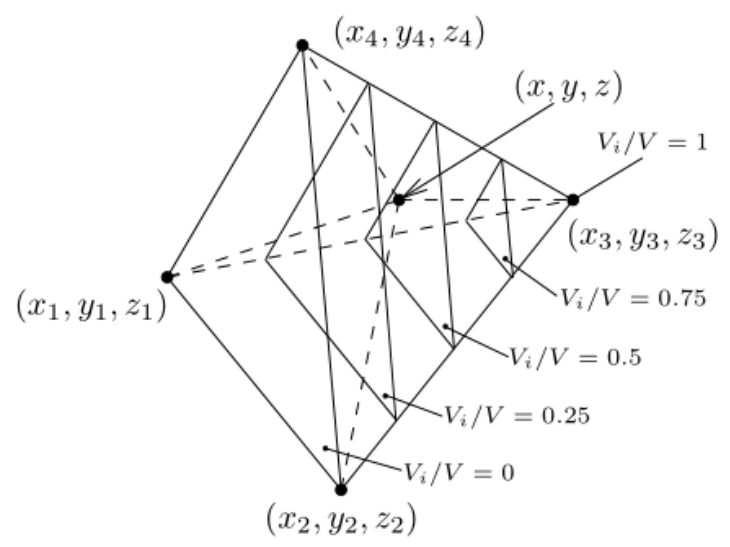

Figure 21. Fraction $V_{i} / V$ measures the perpendicular distance of the point $(x, y, z)$ toward the facet opposite to node $i$ (here $i=3$ ).

The higher order shape functions can be worked out similarly as it was mentioned in the case of triangular elements. The barycentric coordinates $L_{1}, L_{2}, L_{3}$ and $L_{4}$ can be used. A polynomial of order $n$ must contain all possible terms $x^{p} y^{q} z^{r}, 0 \leq p+q+r \leq n$ and a polynomial contains

$$
m=\frac{(n+1)(n+2)(n+3)}{6}
$$

elements altogether, i.e. $m=1, m=4, m=10$ and $m=20$ in the case of zeroth, first, second and third order polynomials. It means that $m$ coefficients must be expressed and $m$ points must be placed within a tetrahedron.

The interpolation function of order $n$ can be constructed as

$$
N_{i}=P_{I}^{n}\left(L_{1}\right) P_{J}^{n}\left(L_{2}\right) P_{K}^{n}\left(L_{3}\right) P_{L}^{n}\left(L_{4}\right), \quad \text { where } \quad I+J+K+L=n,
$$

where the integers $I, J, K$ and $L$ label the nodes within the tetrahedra, resulting in a numbering scheme. Figure 22, Fig. 23 and Fig. 24 illustrate the numbering scheme of the first, the second and the third order approximations.

The polynomials $P_{I}^{n}\left(L_{1}\right), P_{J}^{n}\left(L_{2}\right), P_{K}^{n}\left(L_{3}\right)$ and $P_{L}^{n}\left(L_{4}\right)$ are defined in the same way as it was presented in the $2 \mathrm{D}$ situation, see definitions (22)-(25).

If $n=1$, then $m=4$, i.e. (see Fig. 22)

$$
\begin{aligned}
& N_{1}=P_{1}^{1}\left(L_{1}\right) P_{0}^{1}\left(L_{2}\right) P_{0}^{1}\left(L_{3}\right) P_{0}^{1}\left(L_{4}\right)=L_{1}, \\
& N_{2}=P_{0}^{1}\left(L_{1}\right) P_{1}^{1}\left(L_{2}\right) P_{0}^{1}\left(L_{3}\right) P_{0}^{1}\left(L_{4}\right)=L_{2},
\end{aligned}
$$




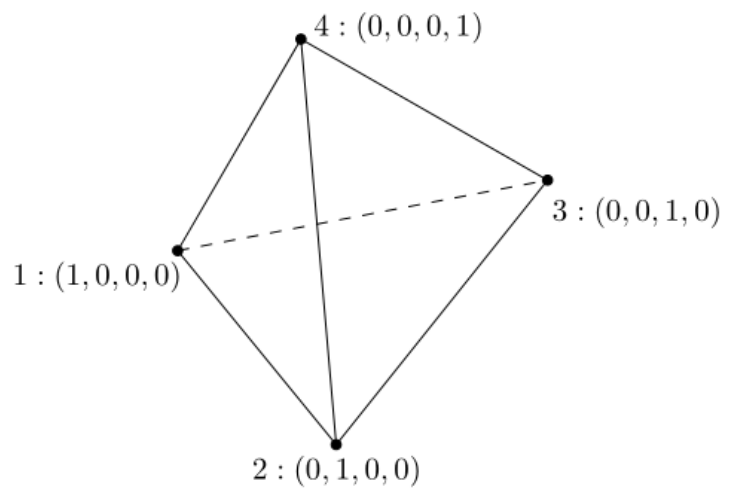

Figure 22. Numbering scheme for linear element, $n=1$.

$$
\begin{aligned}
& N_{3}=P_{0}^{1}\left(L_{1}\right) P_{0}^{1}\left(L_{2}\right) P_{1}^{1}\left(L_{3}\right) P_{0}^{1}\left(L_{4}\right)=L_{3}, \\
& N_{4}=P_{0}^{1}\left(L_{1}\right) P_{0}^{1}\left(L_{2}\right) P_{0}^{1}\left(L_{3}\right) P_{1}^{1}\left(L_{4}\right)=L_{4},
\end{aligned}
$$

since (29) as it was mentioned in (57).

If $n=2$, then $m=10$, i.e. (see Fig. 23)

$$
\begin{aligned}
& N_{1}=P_{2}^{2}\left(L_{1}\right) P_{0}^{2}\left(L_{2}\right) P_{0}^{2}\left(L_{3}\right) P_{0}^{2}\left(L_{4}\right)=L_{1}\left(2 L_{1}-1\right), \\
& N_{2}=P_{0}^{2}\left(L_{1}\right) P_{2}^{2}\left(L_{2}\right) P_{0}^{2}\left(L_{3}\right) P_{0}^{2}\left(L_{4}\right)=L_{2}\left(2 L_{2}-1\right),
\end{aligned}
$$

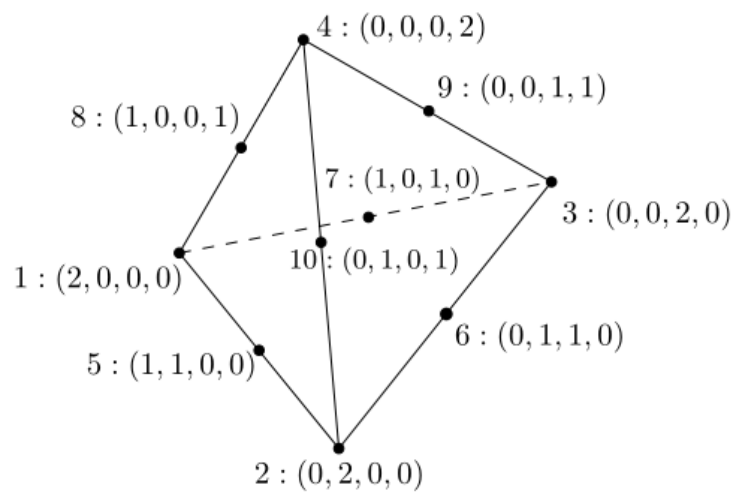

Figure 23. Numbering scheme for quadratic element, $n=2$. 


$$
\begin{gathered}
N_{3}=P_{0}^{2}\left(L_{1}\right) P_{0}^{2}\left(L_{2}\right) P_{2}^{2}\left(L_{3}\right) P_{0}^{2}\left(L_{4}\right)=L_{3}\left(2 L_{3}-1\right), \\
N_{4}=P_{0}^{2}\left(L_{1}\right) P_{0}^{2}\left(L_{2}\right) P_{0}^{2}\left(L_{3}\right) P_{2}^{2}\left(L_{4}\right)=L_{4}\left(2 L_{4}-1\right), \\
N_{5}=P_{1}^{2}\left(L_{1}\right) P_{1}^{2}\left(L_{2}\right) P_{0}^{2}\left(L_{3}\right) P_{0}^{2}\left(L_{4}\right)=4 L_{1} L_{2}, \\
N_{6}=P_{0}^{2}\left(L_{1}\right) P_{1}^{2}\left(L_{2}\right) P_{1}^{2}\left(L_{3}\right) P_{0}^{2}\left(L_{4}\right)=4 L_{2} L_{3}, \\
N_{7}=P_{1}^{2}\left(L_{1}\right) P_{0}^{2}\left(L_{2}\right) P_{1}^{2}\left(L_{3}\right) P_{0}^{2}\left(L_{4}\right)=4 L_{1} L_{3}, \\
N_{8}=P_{1}^{2}\left(L_{1}\right) P_{0}^{2}\left(L_{2}\right) P_{0}^{2}\left(L_{3}\right) P_{1}^{2}\left(L_{4}\right)=4 L_{1} L_{4}, \\
N_{9}=P_{0}^{2}\left(L_{1}\right) P_{0}^{2}\left(L_{2}\right) P_{1}^{2}\left(L_{3}\right) P_{1}^{2}\left(L_{4}\right)=4 L_{3} L_{4}, \\
N_{10}=P_{0}^{2}\left(L_{1}\right) P_{1}^{2}\left(L_{2}\right) P_{0}^{2}\left(L_{3}\right) P_{1}^{2}\left(L_{4}\right)=4 L_{2} L_{4},
\end{gathered}
$$

because (36) and (37).

Finally, if $n=3, m=20$ shape functions can be constructed as (see Fig. 24)

$$
\begin{gathered}
N_{1}=P_{3}^{3}\left(L_{1}\right) P_{0}^{3}\left(L_{2}\right) P_{0}^{3}\left(L_{3}\right) P_{0}^{3}\left(L_{4}\right)=\frac{1}{2} L_{1}\left(3 L_{1}-1\right)\left(3 L_{1}-2\right), \\
N_{2}=P_{0}^{3}\left(L_{1}\right) P_{3}^{3}\left(L_{2}\right) P_{0}^{3}\left(L_{3}\right) P_{0}^{3}\left(L_{4}\right)=\frac{1}{2} L_{2}\left(3 L_{2}-1\right)\left(3 L_{2}-2\right), \\
N_{3}=P_{0}^{3}\left(L_{1}\right) P_{0}^{3}\left(L_{2}\right) P_{3}^{3}\left(L_{3}\right) P_{0}^{3}\left(L_{4}\right)=\frac{1}{2} L_{3}\left(3 L_{3}-1\right)\left(3 L_{3}-2\right), \\
N_{4}=P_{0}^{3}\left(L_{1}\right) P_{0}^{3}\left(L_{2}\right) P_{0}^{3}\left(L_{3}\right) P_{3}^{3}\left(L_{4}\right)=\frac{1}{2} L_{4}\left(3 L_{4}-1\right)\left(3 L_{4}-2\right), \\
N_{5}=P_{2}^{3}\left(L_{1}\right) P_{1}^{3}\left(L_{2}\right) P_{0}^{3}\left(L_{3}\right) P_{0}^{3}\left(L_{4}\right)=\frac{9}{2} L_{1}\left(3 L_{1}-1\right) L_{2},
\end{gathered}
$$

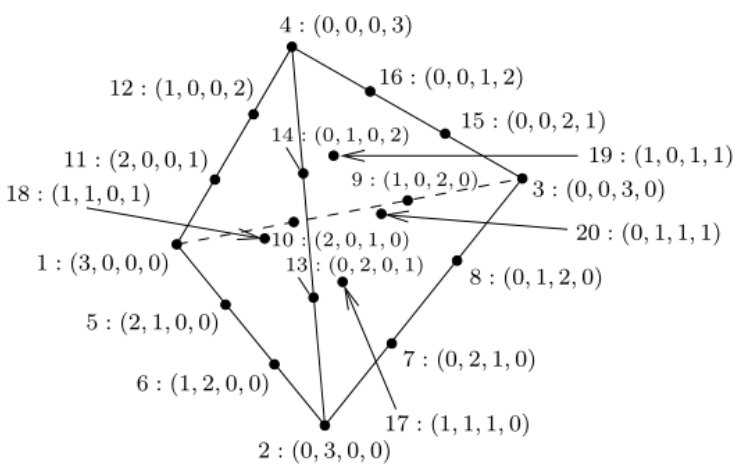

Figure 24. Numbering scheme for cubic element, $n=3$. 


$$
\begin{gathered}
N_{6}=P_{1}^{3}\left(L_{1}\right) P_{2}^{3}\left(L_{2}\right) P_{0}^{3}\left(L_{3}\right) P_{0}^{3}\left(L_{4}\right)=\frac{9}{2} L_{2}\left(3 L_{2}-1\right) L_{1}, \\
N_{7}=P_{0}^{3}\left(L_{1}\right) P_{2}^{3}\left(L_{2}\right) P_{1}^{3}\left(L_{3}\right) P_{0}^{3}\left(L_{4}\right)=\frac{9}{2} L_{2}\left(3 L_{2}-1\right) L_{3}, \\
N_{8}=P_{0}^{3}\left(L_{1}\right) P_{1}^{3}\left(L_{2}\right) P_{2}^{3}\left(L_{3}\right) P_{0}^{3}\left(L_{4}\right)=\frac{9}{2} L_{3}\left(3 L_{3}-1\right) L_{2}, \\
N_{9}=P_{1}^{3}\left(L_{1}\right) P_{0}^{3}\left(L_{2}\right) P_{2}^{3}\left(L_{3}\right) P_{0}^{3}\left(L_{4}\right)=\frac{9}{2} L_{3}\left(3 L_{3}-1\right) L_{1}, \\
N_{10}=P_{2}^{3}\left(L_{1}\right) P_{0}^{3}\left(L_{2}\right) P_{1}^{3}\left(L_{3}\right) P_{0}^{3}\left(L_{4}\right)=\frac{9}{2} L_{1}\left(3 L_{1}-1\right) L_{3}, \\
N_{11}=P_{1}^{3}\left(L_{1}\right) P_{0}^{3}\left(L_{2}\right) P_{0}^{3}\left(L_{3}\right) P_{2}^{3}\left(L_{4}\right)=\frac{9}{2} L_{4}\left(3 L_{4}-1\right) L_{1}, \\
N_{12}=P_{2}^{3}\left(L_{1}\right) P_{0}^{3}\left(L_{2}\right) P_{0}^{3}\left(L_{3}\right) P_{1}^{3}\left(L_{4}\right)=\frac{9}{2} L_{1}\left(3 L_{1}-1\right) L_{4}, \\
N_{13}=P_{0}^{3}\left(L_{1}\right) P_{1}^{3}\left(L_{2}\right) P_{0}^{3}\left(L_{3}\right) P_{2}^{3}\left(L_{4}\right)=\frac{9}{2} L_{4}\left(3 L_{4}-1\right) L_{2}, \\
N_{14}=P_{0}^{3}\left(L_{1}\right) P_{2}^{3}\left(L_{2}\right) P_{0}^{3}\left(L_{3}\right) P_{1}^{3}\left(L_{4}\right)=\frac{9}{2} L_{2}\left(3 L_{2}-1\right) L_{4}, \\
N_{15}=P_{0}^{3}\left(L_{1}\right) P_{0}^{3}\left(L_{2}\right) P_{1}^{3}\left(L_{3}\right) P_{2}^{3}\left(L_{4}\right)=\frac{9}{2} L_{4}\left(3 L_{4}-1\right) L_{3}, \\
N_{18}=P_{1}^{3}\left(L_{1}\right) P_{1}^{3}\left(L_{2}\right) P_{0}^{3}\left(L_{3}\right) P_{1}^{3}\left(L_{4}\right)=27 L_{1} L_{2} L_{4}, \\
N_{20}=P_{0}^{3}\left(L_{1}\right) P_{0}^{3}\left(L_{2}\right) P_{1}^{3}\left(L_{3}\right) P_{1}^{3}\left(L_{4}\right)=27 L_{1} L_{3} L_{4}, \\
N_{1}\left(L_{1}\right) P_{0}^{3}\left(L_{2}\right) P_{2}^{3}\left(L_{3}\right) P_{1}^{3}\left(L_{4}\right)=\frac{9}{2} L_{3}\left(3 L_{3}-1\right) L_{4}, \\
N_{1}\left(L_{1}\right) P_{1}^{3}\left(L_{2}\right) P_{1}^{3}\left(L_{3}\right) P_{0}^{3}\left(L_{4}\right)=27 L_{2} L_{3} L_{4},
\end{gathered}
$$

because of the equations (48), (49) and (50).

The scalar potential along any edge of a tetrahedron is the linear combination of the values defined on the points of the given edge, so that if two tetrahedra share the same facet, the potential will be continuous across this interface. This means that the approximate solution is continuous everywhere, however, its normal derivate is not.

If potentials at the nodes are known, then a linear approximation of the potential function can be represented by (1).

The sum of all nodal shape functions is equal to 1 , hence the sum of their gradient is zero,

$$
\sum_{i=1}^{m} N_{i}=1, \quad \text { and } \quad \sum_{i=1}^{m} \nabla N_{i}=0 .
$$


This means that the maximal number of linearly independent nodal basis functions is $m$ and the maximal number of linearly independent gradients of the nodal basis functions is $m-1$, i.e. shape functions are linearly independent but their gradients are not.

\subsection{Edge finite elements}

Vector potentials can be represented either by nodal shape functions or by so-called edge shape functions. Edge shape functions are also called vector shape functions.

The natural approach is to treat the vector field $\boldsymbol{T}=\boldsymbol{T}(\boldsymbol{r}, t)$ as two or three coupled scalar fields $T_{x}=T_{x}(\boldsymbol{r}, t), T_{y}=T_{y}(\boldsymbol{r}, t)$ and $T_{z}=T_{z}(\boldsymbol{r}, t)$, i.e.

$$
\boldsymbol{T}=T_{x} \boldsymbol{e}_{x}+T_{y} \boldsymbol{e}_{y}
$$

and

$$
\boldsymbol{T}=T_{x} \boldsymbol{e}_{x}+T_{y} \boldsymbol{e}_{y}+T_{z} \boldsymbol{e}_{z}
$$

in 2D and in 3D situations, respectively, $\boldsymbol{e}_{x}, \boldsymbol{e}_{y}$ and $\boldsymbol{e}_{z}$ are the orthogonal unit vectors in the $x-y$ and in the $x-y-z$ plane.

Nodal shape functions can be used in this case as well, as it was presented for scalar potentials in the previous section, however, each node has two or three unknowns. Nodal shape functions can be applied to approximate the scalar components of the vector field $\boldsymbol{T}$. For example in 3D, $\boldsymbol{T}$ can be approximated as

$$
\begin{aligned}
\boldsymbol{T} & \simeq \sum_{i=1}^{m} N_{i}\left(T_{x, i} \boldsymbol{e}_{x}+T_{y, i} \boldsymbol{e}_{y}+T_{z, i} \boldsymbol{e}_{z}\right) \\
& =\sum_{i=1}^{m} N_{i} T_{x, i} \boldsymbol{e}_{x}+\sum_{i=1}^{m} N_{i} T_{y, i} \boldsymbol{e}_{y}+\sum_{i=1}^{m} N_{i} T_{z, i} \boldsymbol{e}_{z} .
\end{aligned}
$$

Here $N_{i}=N_{i}(\boldsymbol{r})$ are the usual nodal shape functions defined by (2) and $T_{x, i}=T_{x, i}(t)$, $T_{y, i}=T_{y, i}(t), T_{z, i}=T_{z, i}(t)$ are the values of components of the approximated vector potential at node $i$. The number of degrees of freedom is $2 m$ in a $2 \mathrm{D}$ problem using triangular mesh and $3 m$ in a $3 \mathrm{D}$ arrangement meshed by tetrahedral elements.

Nodal shape functions are used to approximate gauged vector potentials, which was the first in the history of finite element method in electromagnetics. Unfortunately, there are some problems when the usual nodal based finite elements are used to interpolate vector potentials. The lack of enforcement of the divergence condition (lack of gauging) results in a system of algebraic equations, which has infinite number of solution and the application of iterative solvers sometimes fails. We have to take care about the Coulomb gauge. There are problems on the iron/air interface when using the magnetic vector potential approximated by nodal elements and extra interface conditions must be set up to solve this problem. 
Fortunately, vector shape functions have been developed in the last decades, which application in static and eddy current field problems is more and more popular, because of their advantages. The use of edge shape functions solves the problems described above. It will be shown that the divergence of vector shape functions is equal to zero, that is why, gauging is satisfied automatically. The ungauged potential functions are approximated by vector elements. Vector shape functions are usually called edge shape functions, because they are associated to the edges of the FEM mesh. Vector shape functions are more and more popular in wave problems, too.

Instead of scalar shape functions, vector shape functions (or edge shape functions) $\boldsymbol{W}_{i}=\boldsymbol{W}_{i}(\boldsymbol{r})$ can be applied to approximate a vector potential $\boldsymbol{T}$,

$$
\boldsymbol{T} \simeq \sum_{i=1}^{k} \boldsymbol{W}_{i} T_{i}
$$

where $T_{i}=T_{i}(t)$ is the line integral of the vector potential $\boldsymbol{T}$ along the edge $i$. First order vector shape functions are defined by the line integral

$$
\int_{l} \boldsymbol{W}_{i} \cdot \mathrm{d} \boldsymbol{l}= \begin{cases}1, & \text { along edge } i, \\ 0, & \text { along other edges, }\end{cases}
$$

i.e. the line integral of the vector shape function $\boldsymbol{W}_{i}$ along the $i^{\text {th }}$ edge is equal to one. In other words, the vector shape function $\boldsymbol{W}_{i}$ has tangential component only along the $i^{\text {th }}$ edge and it has only normal component along the other edges, because $\boldsymbol{W}_{i} \cdot \mathrm{d} \boldsymbol{l}$ is equal to zero only if the vectors $\boldsymbol{W}_{i}$ and $\mathrm{d} \boldsymbol{l}$ are perpendicular to each others and $\left|\boldsymbol{W}_{i}\right||\mathrm{d} \boldsymbol{l}|>0$. Moreover, in 3D case, the vector shape function $\boldsymbol{W}_{i}$ has zero tangential component along every facet of the 3D finite element, which not share the edge $i$.

If two triangles share the same vertices, the tangential component of the approximated vector potential will be continuous across the interface element boundary. This is true in $3 \mathrm{D}$ case as well, moreover, if two tetrahedra share the same facet, the tangential component of the vector potential will be continuous across this interface. This means that the tangential component of the approximate solution is continuous everywhere, however, its normal component is not. In the words of equations, according to the definition (99), the line integral of the vector potential along the $m^{\text {th }}$ edge is equal to $T_{m}$, i.e.

$$
\begin{aligned}
\int_{l_{m}} \boldsymbol{T} \cdot \mathrm{d} \boldsymbol{l} & =\int_{l_{m}}\left(\sum_{i=1}^{k} \boldsymbol{W}_{i} T_{i}\right) \cdot \mathrm{d} \boldsymbol{l}=\sum_{i=1}^{k} \int_{l_{m}}\left(\boldsymbol{W}_{i} T_{i}\right) \cdot \mathrm{d} \boldsymbol{l} \\
& =T_{m} \int_{l_{m}} \boldsymbol{W}_{m} \cdot \mathrm{d} \boldsymbol{l}=T_{m} .
\end{aligned}
$$


That is why, edge shape functions are also called tangentially continuous shape functions.

The gradients of the nodal shape functions are in the function space spanned by the edge basis functions, that is

$$
\nabla N_{j}=\sum_{i=1}^{k} c_{j i} \boldsymbol{W}_{i}, \quad j=1, \cdots, m-1,
$$

where $\sum_{i=1}^{k} c_{j i}^{2}>0$. Taking the curl of each equation in (101) results in

$$
\sum_{i=1}^{k} c_{j i} \nabla \times \boldsymbol{W}_{i}=\mathbf{0}, \quad j=1, \cdots, m-1,
$$

because $\nabla \times(\nabla \varphi) \equiv 0$. This shows that the maximal number of linearly independent curls of the edge basis functions is $k-(m-1)$. The interdependence of the curls of the edge basis functions means that an ungauged formulation leads to a singular, positive semidefinit finite element curl-curl matrix. Singular systems can be solved by iterative methods, if the right-hand side of the system of equations is consistent. We took care about it when obtaining the weak formulations of the ungauged version of potentials, because excitation current density has been taken into account by the use of impressed current vector potential, $\boldsymbol{T}_{0}$.

The vector function

$$
\boldsymbol{w}_{i j}=L_{i} \nabla L_{j}-L_{j} \nabla L_{i}
$$

will be applied to construct the edge shape functions, because it can be used in functions, which satisfies (99) and (100). In 2D, $L_{i}(i=1,2,3)$ are the barycentric coordinates of the triangle defined by (18). In $3 \mathrm{D}, L_{i}(i=1,2,3,4)$ are the barycentric coordinates of

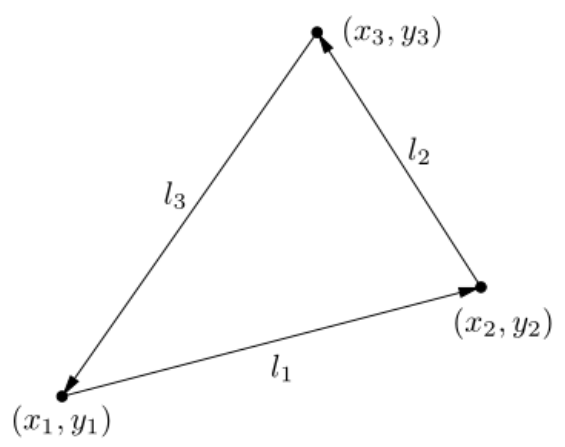

Figure 25. The definition of edges with local directions of the triangular finite element. 


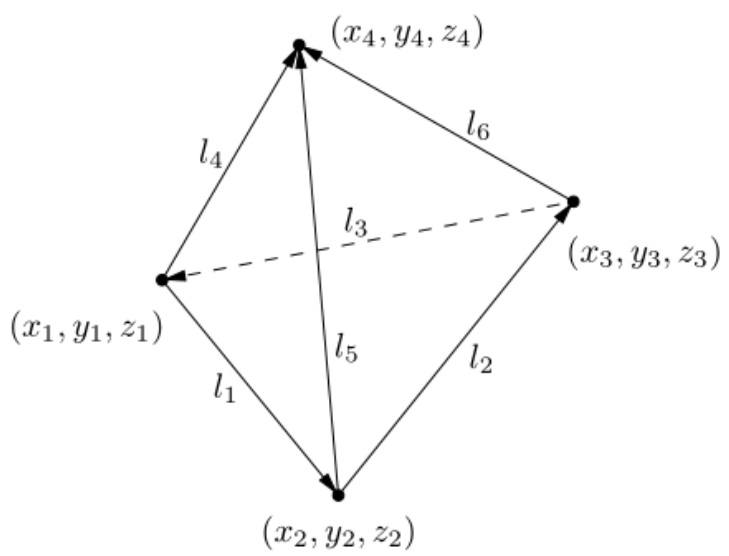

Figure 26. The definition of edges with local directions of the tetrahedral finite element.

the tetrahedron defined by (56). According to the notations in (103), the edges of a finite element are pointing from node $i$ to node $j$, as it can be seen in Fig. 25 and in Fig. 26.

The vector field $\boldsymbol{w}_{i j}$ has the following important properties, which proofs the use of vector function $\boldsymbol{w}_{i j}$ as vector shape function.

(i) Let $\boldsymbol{e}_{i j}$ is a unit vector pointing from node $i$ to node $j$, then

$$
\boldsymbol{e}_{i j} \cdot \boldsymbol{w}_{i j}=\frac{1}{l_{i j}},
$$

where $l_{i j}$ is the length of edge $\{i, j\}$. This means that $\boldsymbol{w}_{i j}$ has constant tangential component along the edge $\{i, j\}$.

Since $L_{i}$ and $L_{j}$ are linear functions that vary from node $i$ to node $j$ from 1 to 0 and from 0 to 1 , respectively, we have $\boldsymbol{e}_{i j} \cdot \nabla L_{i}=-1 / l_{i j}$ and $\boldsymbol{e}_{i j} \cdot \nabla L_{j}=1 / l_{i j}$, finally

$$
\boldsymbol{e}_{i j} \cdot \boldsymbol{w}_{i j}=L_{i} \frac{1}{l_{i j}}+L_{j} \frac{1}{l_{i j}}=\frac{L_{i}+L_{j}}{l_{i j}}=\frac{1}{l_{i j}},
$$

because $L_{i}+L_{j}=1$ along the edge $\{i, j\}$. See, for example Fig. 14 and let $i=1$, $j=2$, so $N_{1}=L_{1}$ is decreasing along edge $\{1,2\}$ and $N_{2}=L_{2}$ is increasing along the same edge. See also Fig. 7, from which it is easy to see the gradients $\boldsymbol{e}_{i j} \cdot \nabla L_{i}=-1 / l_{i j}$ and $\boldsymbol{e}_{i j} \cdot \nabla L_{j}=1 / l_{i j}$. 
(ii) In 2D, the function $L_{i}$ varies linearly from node $i$ to the opposite edge $\{j, k\}$ (see e.g. $N_{1}$ in Fig. 14, $i=1, j=2, k=3$ ), i.e. the vector field $\nabla L_{i}$ is perpendicular to this edge, but $L_{i}$ is zero there, that is why $\boldsymbol{w}_{i j}$ is perpendicular to the edge $\{j, k\}$,

$$
\boldsymbol{w}_{i j}=-L_{j} \nabla L_{i}, \quad \text { on the edge }\{j, k\},
$$

and the length of this vector is decreasing from node $j$ to $k$ according to $L_{j}$. On the other hand, the function $L_{j}$ varies linearly from node $j$ to the opposite edge $\{k, i\}$ (see $N_{2}$ in Fig. 14), i.e. $\nabla L_{j}$ is perpendicular to this edge, but $L_{j}$ is zero there and $\boldsymbol{w}_{i j}$ is perpendicular to the edge $\{k, i\}$,

$$
\boldsymbol{w}_{i j}=L_{i} \nabla L_{j}, \quad \text { on the edge } \quad\{k, i\},
$$

and the length of this vector is decreasing from node $i$ to $k$ according to $L_{i}$.

This with item (i) means that the vector function $\boldsymbol{w}_{i j}$ has tangential component only on the edge $\{i, j\}$ and it is perpendicular to the other edges.

In $3 \mathrm{D}$, this is valid to the whole triangular facet with the bounding edges opposite to a node, see e.g. Fig. 21.

(iii) The vector field $\boldsymbol{w}_{i j}$ is divergence-free,

$$
\begin{aligned}
\nabla \cdot \boldsymbol{w}_{i j} & =\nabla \cdot\left(L_{i} \nabla L_{j}-L_{j} \nabla L_{i}\right)=\nabla \cdot\left(L_{i} \nabla L_{j}\right)-\nabla \cdot\left(L_{j} \nabla L_{i}\right) \\
& =\nabla L_{i} \cdot \nabla L_{j}+L_{i} \nabla \cdot \nabla L_{j}-\nabla L_{j} \cdot \nabla L_{i}-L_{j} \nabla \cdot \nabla L_{i}=0,
\end{aligned}
$$

by using the identity

$$
\nabla \cdot(\varphi \boldsymbol{v})=\nabla \varphi \cdot \boldsymbol{v}+\varphi \nabla \cdot \boldsymbol{v}
$$

with the notations $\varphi=L_{i}, \boldsymbol{v}=\nabla L_{j}$ in the second and $\varphi=L_{j}, \boldsymbol{v}=\nabla L_{i}$ in the last term. The barycentric coordinates are linear functions of the coordinates and their gradient is constant, which divergence is equal to zero, i.e. the second and fourth terms are vanishing. The first and the third terms are equal, finally, $\nabla \cdot \boldsymbol{w}_{i j}=0$.

(iv) The vector field $\boldsymbol{w}_{i j}$ has constant curl,

$$
\begin{aligned}
\nabla \times \boldsymbol{w}_{i j} & =\nabla \times\left(L_{i} \nabla L_{j}-L_{j} \nabla L_{i}\right)=\nabla \cdot\left(L_{i} \nabla L_{j}\right)-\nabla \cdot\left(L_{j} \nabla L_{i}\right) \\
& =L_{i} \nabla \times \nabla L_{j}-\nabla L_{j} \times \nabla L_{i}-L_{j} \nabla \times \nabla L_{i}+\nabla L_{i} \times \nabla L_{j} \\
& =2 \nabla L_{i} \times \nabla L_{j},
\end{aligned}
$$

by using the identity 


$$
\nabla \times(\varphi \boldsymbol{v})=\varphi \nabla \times \boldsymbol{v}-\boldsymbol{v} \times \nabla \varphi
$$

with the notations $\varphi=L_{i}, \boldsymbol{v}=\nabla L_{j}$ in the first and $\varphi=L_{j}, \boldsymbol{v}=\nabla L_{i}$ in the second term. The first and the third terms are equal to zero because of the identity $\nabla \times \nabla \varphi \equiv 0$ for any function $\varphi$. The second term can be reformulated by $\boldsymbol{a} \times \boldsymbol{b}=-\boldsymbol{b} \times \boldsymbol{a}$, finally, the result is constant, because the gradients of the barycentric coordinates are constant.

First, the edge shape functions defined on triangles based on (103) are collected. The basic $2 \mathrm{D}$ vector shape functions $\boldsymbol{W}_{i}$ can be constructed by using the first order nodal shape functions,

$$
\begin{aligned}
& \boldsymbol{W}_{1}=l_{1}\left(N_{1} \nabla N_{2}-N_{2} \nabla N_{1}\right) \delta_{1}, \\
& \boldsymbol{W}_{2}=l_{2}\left(N_{2} \nabla N_{3}-N_{3} \nabla N_{2}\right) \delta_{2}, \\
& \boldsymbol{W}_{3}=l_{3}\left(N_{3} \nabla N_{1}-N_{1} \nabla N_{3}\right) \delta_{3} .
\end{aligned}
$$

Here $l_{i}$ (Fig. 25) denotes the length of the $i^{\text {th }}$ edges of the triangle and it is used to normalize the edge shape function according to (104). The edge basis function $\boldsymbol{W}_{i}(i=1,2,3)$ has tangential component only along the $i^{\text {th }}$ edge and it is perpendicular to the other two edges as represented in Fig. 27(a)-27(c). It is easy to see that an edge shape function has magnitude and direction. The value of $\delta_{i}$ is equal to \pm 1 , depending on whether the local direction of the edge is the same as the global direction or opposite (see Fig. 25 for local direction). This set of vector functions is called zeroth order vector shape functions.

If the approximation of the vector function $T$ is known along the edges of the mesh, then (98) can be used to interpolate the function anywhere and in linear case $K=3$.

Higher order vector shape functions can be constructed by using the vector function $\boldsymbol{w}_{i j}$ defined by (103), too. This vector function must be multiplied by a complete interpolatory polynomial, which results in the higher order vector shape functions. First and second order polynomials will be used to build up first and second order vector shape functions. Here, we follow, the method is as follows.

First of all, an indexing sequence must be set up, which is similar to the method used to build up the scalar shape functions, because the higher order vector shape functions are based on the Lagrange polynomials and (103). In the case of first order approximation, the numbering scheme of the third order scalar interpolation can be used and the points are shown in Fig. 28, Fig. 29 and Fig. 30 must be used to represent first order vector shape functions associated to the edge $\{1,2\},\{2,3\}$ and $\{3,1\}$, respectively. In the case of second order approximation, the numbering scheme of the fourth order scalar interpolation can be used and the interpolation points shown in Fig. 31, Fig. 32 and Fig. 33 must be used to represent second order vector shape functions associated to the edge $\{1,2\},\{2,3\}$ and $\{3,1\}$, respectively. The interpolation points have been selected in this special way, because the interpolation of field vectors along vertices has been avoided, i.e. the points have been shifted inside the triangle and the indexing scheme of order $n+2$ is used to represent the vector interpolation of order $n$. This is called global numbering and denoted 
M. Kuczmann - Acta Technica Jaurinensis, Vol.8., No.4., pp. 347-383, 2015

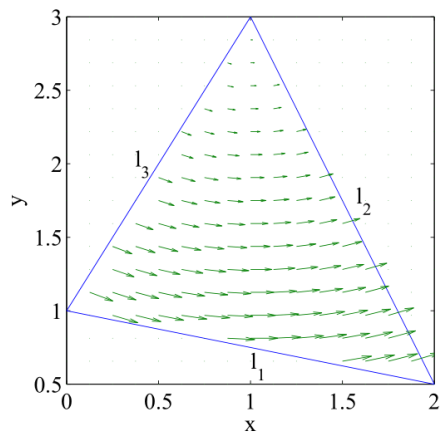

(a) The edge shape function $\boldsymbol{W}_{1}$

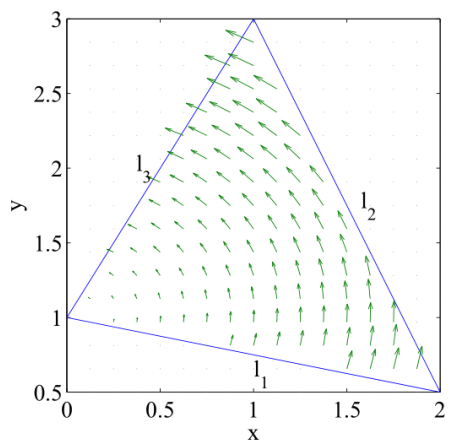

(b) The edge shape function $\boldsymbol{W}_{2}$

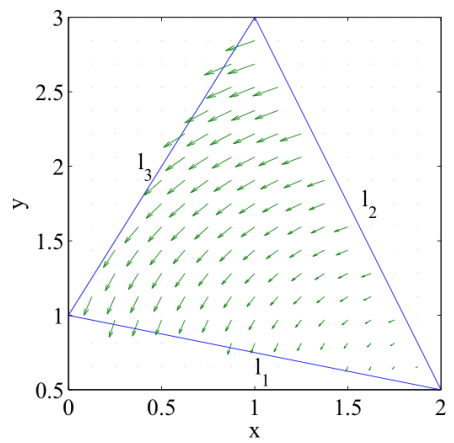

(c) The edge shape function $\boldsymbol{W}_{3}$

Figure 27. The 2D edge shape functions. 


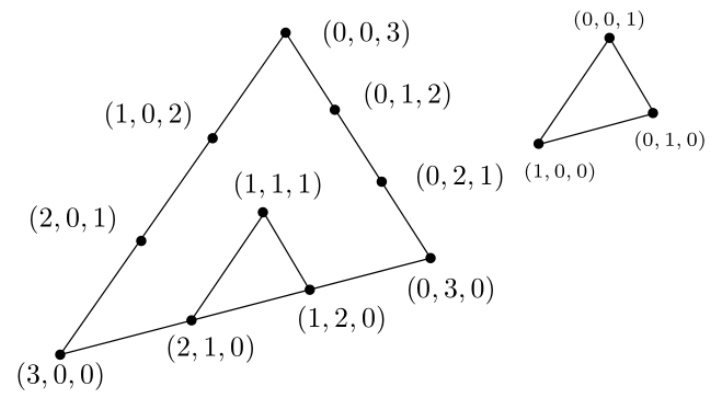

Figure 28. Numbering scheme for the first order vector element associated with $\boldsymbol{w}_{12}$.

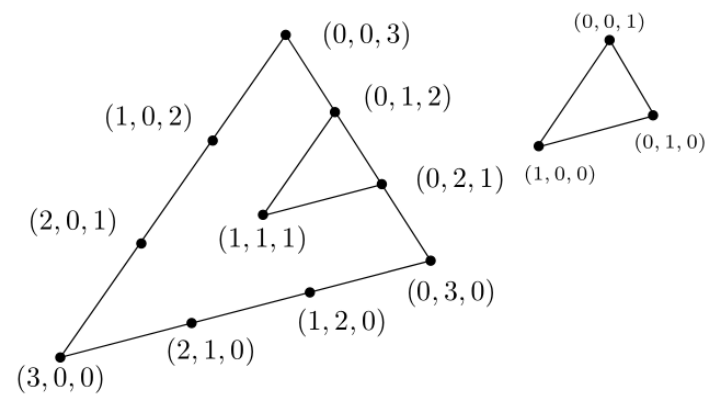

Figure 29. Numbering scheme for the first order vector element associated with $\boldsymbol{w}_{23}$.

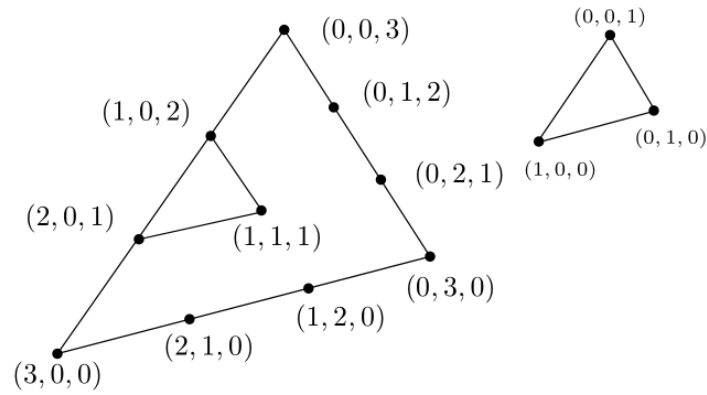

Figure 30. Numbering scheme for the first order vector element associated with $\boldsymbol{w}_{31}$.

by $(I, J, K)$ on the 'big' triangle, local numbering means the numbering scheme with the real order $(i, j, k)$ defined over the 'small' triangle. 


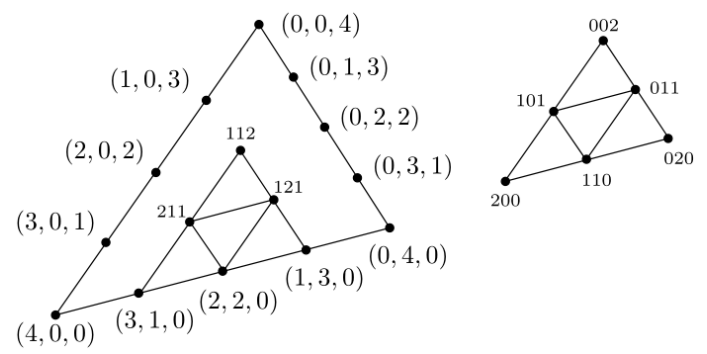

Figure 31. Numbering scheme for the second order vector element associated with $\boldsymbol{w}_{12}$.

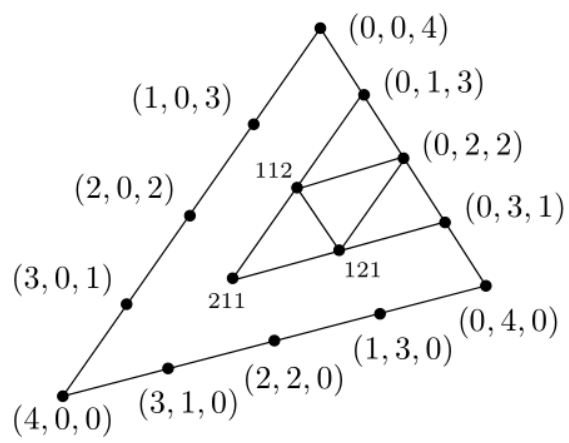

Figure 32. Numbering scheme for the second order vector element associated with $\boldsymbol{w}_{23}$.

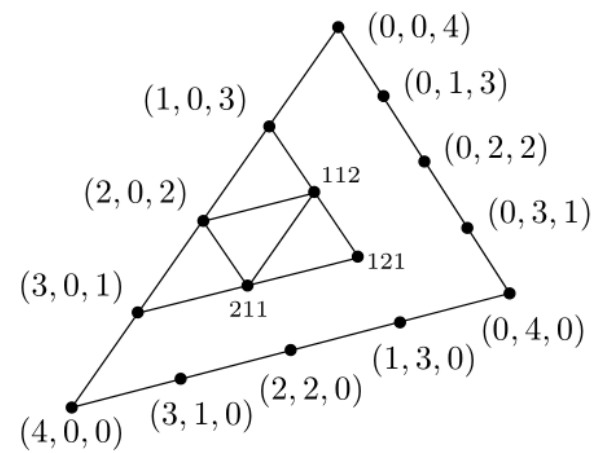

Figure 33. Numbering scheme for the second order vector element associated with $\boldsymbol{w}_{31}$.

It is noted here that the COMSOL Multiphysics software uses this kind of vector shape functions, however, $n=0, n=1$ and $n=2$ are named as linear, quadratic and cubic vector shape functions. 
The vector function $\boldsymbol{w}_{a b}$ (associated to the edge pointing from node $a$ to node $b$ ) can be multiplied by the Lagrange polynomials as

$$
\boldsymbol{W}_{a b}^{I J K}=\alpha_{a b}^{I J K} P_{i}^{n}\left(l_{1}\right) P_{j}^{n}\left(l_{2}\right) P_{k}^{n}\left(l_{3}\right) \boldsymbol{w}_{a b},
$$

where $n$ is the order of approximation and the integers $i, j$ and $k$ satisfy $i+j+k=n$ (see the small triangles in Fig. 28-Fig. 33).

If $n=0$, the basic vector shape functions can be obtained, because $P_{m}^{0}(\cdot)=1$ and $\alpha$ can be selected as the length of the appropriate edge, $l_{a b}$, since $\alpha_{a b}^{I J K}$ is a normalizing factor. The barycentric coordinates $l_{1}, l_{2}$ and $l_{3}$ are imagined in the small triangles. The transformation between local and global numbering is as follows:

$$
\begin{aligned}
& i=I-1, \quad j=J-1, \quad k=K, \quad \text { on the edge }\{1,2\}, \\
& i=I, \quad j=J-1, \quad k=K-1, \quad \text { on the edge }\{2,3\}, \\
& i=I-1, \quad j=J, \quad k=K-1, \quad \text { on the edge }\{3,1\} .
\end{aligned}
$$

The relation between the barycentric coordinates of the small and the big triangles is as follows:

$$
l_{1}=L_{1} \frac{n+2}{n}, \quad l_{2}=L_{2} \frac{n+2}{n}-\frac{1}{n}, \quad l_{3}=L_{3} \frac{n+2}{n}-\frac{1}{n} .
$$

Using these relations, (115) can be written as (let here $\{a b\}=\{23\}$ for simplicity)

$$
\begin{array}{r}
\boldsymbol{W}_{23}^{I J K}=\alpha_{23}^{I J K} P_{I}^{n}\left(L_{1} \frac{n+2}{n}\right) P_{J-1}^{n}\left(L_{2} \frac{n+2}{n}-\frac{1}{n}\right) \\
P_{K-1}^{n}\left(L_{3} \frac{n+2}{n}-\frac{1}{n}\right) \boldsymbol{w}_{23} .
\end{array}
$$

According to (22), Lagrange polynomials can be reformulated as

$$
\begin{aligned}
P_{I}^{n}\left(L_{1} \frac{n+2}{n}\right) & =\frac{1}{I !} \prod_{p=0}^{I-1}\left(n L_{1} \frac{n+2}{n}-p\right) \\
& =\frac{1}{I !} \prod_{p=0}^{I-1}\left[(n+2) L_{1}-p\right]=P_{I}^{n+2}\left(L_{1}\right), \quad \text { if } \quad I>0
\end{aligned}
$$


and

$$
\begin{aligned}
P_{J-1}^{n}\left(L_{2} \frac{n+2}{n}-\frac{1}{n}\right) & =\frac{1}{(J-1) !} \prod_{p=0}^{J-2}\left[n\left(L_{2} \frac{n+2}{n}-\frac{1}{n}\right)-p\right] \\
& =\frac{1}{(J-1) !} \prod_{p=0}^{J-2}\left[(n+2) L_{2}-1-p\right] \\
& =\frac{1}{(J-1) !} \prod_{p=0}^{J-2}\left[(n+2)\left(L_{2}-\frac{1}{n+2}\right)-p\right] \\
& =P_{J-1}^{n+2}\left(L_{2}-\frac{1}{n+2}\right), \text { if } \quad J>1 .
\end{aligned}
$$

The so-called shifted Silvester polynomials can be used to simplify the relations above,

$$
S_{J}^{n+2}\left(L_{2}\right)=P_{J-1}^{n+2}\left(L_{2}-\frac{1}{n+2}\right)=\frac{1}{(J-1) !} \prod_{p=0}^{J-1}\left[(n+2) L_{2}-p\right] .
$$

Finally, the higher order vector shape functions can be formulated as follows by using the Lagrange and Silvester polynomials:

$$
\begin{aligned}
& \boldsymbol{W}_{12}^{I J K}=\alpha_{12}^{I J K} S_{I}^{n+2}\left(L_{1}\right) S_{J}^{n+2}\left(L_{2}\right) P_{K}^{n+2}\left(L_{3}\right) \boldsymbol{w}_{12}, \\
& \boldsymbol{W}_{23}^{I J K}=\alpha_{23}^{I J K} P_{I}^{n+2}\left(L_{1}\right) S_{J}^{n+2}\left(L_{2}\right) S_{K}^{n+2}\left(L_{3}\right) \boldsymbol{w}_{23}, \\
& \boldsymbol{W}_{31}^{I J K}=\alpha_{31}^{I J K} S_{I}^{n+2}\left(L_{1}\right) P_{J}^{n+2}\left(L_{2}\right) S_{K}^{n+2}\left(L_{3}\right) \boldsymbol{w}_{31} .
\end{aligned}
$$

The parameter denoted by $\alpha$ is a normalization factor, which must have the value such that the line integral of vector shape function $\boldsymbol{W}_{a b}^{I J K L}$ is equal to 1 on the edge pointing from node $a$ to node $b$. Here,

$$
P_{0}^{n}(\cdot)=1, \quad \text { and } \quad S_{1}^{n}(\cdot)=1 .
$$

The number of vector basis functions is

$$
k=(n+1)(n+3) .
$$

There is one shape function associated with the introduced interpolation nodes on the edges. It means $3(n+1)$ basis functions. There are three basis functions for an interior interpolation point, because every interpolation point inside the triangle is used to build all the vector shape functions in the three edges. Since a surface vector has only two degrees of freedom, these three basis functions are not independent and one of them must be discarded. This results in $n(n+1)$ interior basis functions. In total, the number of shape functions is $3(n+1)+n(n+1)=(n+3)(n+1)$. 
In the case of first order approximation $n=0$ and $k=3$. In the case of second order approximation $n=1$ and $K=8$. In the case of third order approximation $n=2$ and $k=15$ and so on.

The first order vector shape functions are as follows:

$$
\begin{gathered}
\boldsymbol{W}_{1}=\boldsymbol{W}_{12}^{120}=\alpha_{12}^{120} S_{1}^{3}\left(L_{1}\right) S_{2}^{3}\left(L_{2}\right) P_{0}^{3}\left(L_{3}\right) \boldsymbol{w}_{12}=\alpha_{12}^{120}\left(3 L_{2}-1\right) \boldsymbol{w}_{12}, \\
\boldsymbol{W}_{2}=\boldsymbol{W}_{12}^{210}=\alpha_{12}^{210} S_{2}^{3}\left(L_{1}\right) S_{1}^{3}\left(L_{2}\right) P_{0}^{3}\left(L_{3}\right) \boldsymbol{w}_{12}=\alpha_{12}^{210}\left(3 L_{1}-1\right) \boldsymbol{w}_{12}, \\
\boldsymbol{W}_{3}=\boldsymbol{W}_{12}^{111}=\alpha_{12}^{111} S_{1}^{3}\left(L_{1}\right) S_{1}^{3}\left(L_{2}\right) P_{1}^{3}\left(L_{3}\right) \boldsymbol{w}_{12}=\alpha_{12}^{111} 3 L_{3} \boldsymbol{w}_{12}, \\
\boldsymbol{W}_{4}=\boldsymbol{W}_{23}^{012}=\alpha_{23}^{012} P_{0}^{3}\left(L_{1}\right) S_{1}^{3}\left(L_{2}\right) S_{2}^{3}\left(L_{3}\right) \boldsymbol{w}_{23}=\alpha_{23}^{012}\left(3 L_{3}-1\right) \boldsymbol{w}_{23}, \\
\boldsymbol{W}_{5}=\boldsymbol{W}_{23}^{021}=\alpha_{23}^{021} P_{0}^{3}\left(L_{1}\right) S_{2}^{3}\left(L_{2}\right) S_{1}^{3}\left(L_{3}\right) \boldsymbol{w}_{23}=\alpha_{23}^{021}\left(3 L_{2}-1\right) \boldsymbol{w}_{23}, \\
\boldsymbol{W}_{6}=\boldsymbol{W}_{31}^{102}=\alpha_{31}^{102} S_{1}^{3}\left(L_{1}\right) P_{0}^{3}\left(L_{2}\right) S_{2}^{3}\left(L_{3}\right) \boldsymbol{w}_{31}=\alpha_{31}^{102}\left(3 L_{3}-1\right) \boldsymbol{w}_{31}, \\
\boldsymbol{W}_{7}=\boldsymbol{W}_{31}^{201}=\alpha_{31}^{201} S_{2}^{3}\left(L_{1}\right) P_{0}^{3}\left(L_{2}\right) S_{1}^{3}\left(L_{3}\right) \boldsymbol{w}_{31}=\alpha_{31}^{201}\left(3 L_{1}-1\right) \boldsymbol{w}_{31}, \\
\boldsymbol{W}_{8}=\boldsymbol{W}_{31}^{111}=\alpha_{31}^{111} S_{1}^{3}\left(L_{1}\right) P_{1}^{3}\left(L_{2}\right) S_{1}^{3}\left(L_{3}\right) \boldsymbol{w}_{31}=\alpha_{31}^{111} 3 L_{2} \boldsymbol{w}_{31} .
\end{gathered}
$$

The second order vector shape functions are as follows using (124)-(127):

$$
\begin{aligned}
\boldsymbol{W}_{1}=\boldsymbol{W}_{12}^{310} & =\alpha_{12}^{310} S_{3}^{4}\left(L_{1}\right) S_{1}^{4}\left(L_{2}\right) P_{0}^{4}\left(L_{3}\right) \boldsymbol{w}_{12} \\
& =\alpha_{12}^{310} \frac{1}{2}\left(4 L_{1}-1\right)\left(4 L_{1}-2\right) \boldsymbol{w}_{12}, \\
\boldsymbol{W}_{2}=\boldsymbol{W}_{12}^{220} & =\alpha_{12}^{220} S_{2}^{4}\left(L_{1}\right) S_{2}^{4}\left(L_{2}\right) P_{0}^{4}\left(L_{3}\right) \boldsymbol{w}_{12} \\
& =\alpha_{12}^{220}\left(4 L_{1}-1\right)\left(4 L_{2}-1\right) \boldsymbol{w}_{12}, \\
\boldsymbol{W}_{3}=\boldsymbol{W}_{12}^{130} & =\alpha_{12}^{130} S_{1}^{4}\left(L_{1}\right) S_{3}^{4}\left(L_{2}\right) P_{0}^{4}\left(L_{3}\right) \boldsymbol{w}_{12} \\
& =\alpha_{12}^{130} \frac{1}{2}\left(4 L_{2}-1\right)\left(4 L_{2}-2\right) \boldsymbol{w}_{12}, \\
\boldsymbol{W}_{4}=\boldsymbol{W}_{12}^{211} & =\alpha_{12}^{211} S_{2}^{4}\left(L_{1}\right) S_{1}^{4}\left(L_{2}\right) P_{1}^{4}\left(L_{3}\right) \boldsymbol{w}_{12} \\
& =\alpha_{12}^{211}\left(4 L_{1}-1\right) 4 L_{3} \boldsymbol{w}_{12}, \\
\boldsymbol{W}_{5}=\boldsymbol{W}_{12}^{121} & =\alpha_{12}^{121} S_{1}^{4}\left(L_{1}\right) S_{2}^{4}\left(L_{2}\right) P_{1}^{4}\left(L_{3}\right) \boldsymbol{w}_{12} \\
& =\alpha_{12}^{121}\left(4 L_{2}-1\right) 4 L_{3} \boldsymbol{w}_{12}, \\
\boldsymbol{W}_{6}=\boldsymbol{W}_{23}^{031} & =\alpha_{23}^{031} P_{0}^{4}\left(L_{1}\right) S_{3}^{4}\left(L_{2}\right) S_{1}^{4}\left(L_{3}\right) \boldsymbol{w}_{23} \\
& =\alpha_{23}^{031} \frac{1}{2}\left(4 L_{2}-1\right)\left(4 L_{2}-2\right) \boldsymbol{w}_{23}, \\
\boldsymbol{W}_{7}=\boldsymbol{W}_{23}^{022} & =\alpha_{23}^{022} P_{0}^{4}\left(L_{1}\right) S_{2}^{4}\left(L_{2}\right) S_{2}^{4}\left(L_{3}\right) \boldsymbol{w}_{23} \\
& =\alpha_{23}^{022}\left(4 L_{2}-1\right)\left(4 L_{3}-1\right) \boldsymbol{w}_{23},
\end{aligned}
$$




$$
\begin{aligned}
\boldsymbol{W}_{8}=\boldsymbol{W}_{23}^{013} & =\alpha_{23}^{013} P_{0}^{4}\left(L_{1}\right) S_{1}^{4}\left(L_{2}\right) S_{3}^{4}\left(L_{3}\right) \boldsymbol{w}_{23} \\
& =\alpha_{23}^{013} \frac{1}{2}\left(4 L_{3}-1\right)\left(4 L_{3}-2\right) \boldsymbol{w}_{23}, \\
\boldsymbol{W}_{9}=\boldsymbol{W}_{23}^{121} & =\alpha_{23}^{121} P_{1}^{4}\left(L_{1}\right) S_{2}^{4}\left(L_{2}\right) S_{1}^{4}\left(L_{3}\right) \boldsymbol{w}_{23} \\
& =\alpha_{23}^{121}\left(4 L_{1}\right)\left(4 L_{2}-1\right) \boldsymbol{w}_{23}, \\
\boldsymbol{W}_{10}=\boldsymbol{W}_{23}^{112} & =\alpha_{23}^{112} P_{1}^{4}\left(L_{1}\right) S_{1}^{4}\left(L_{2}\right) S_{2}^{4}\left(L_{3}\right) \boldsymbol{w}_{23} \\
& =\alpha_{23}^{112}\left(4 L_{1}\right)\left(4 L_{3}-1\right) \boldsymbol{w}_{23}, \\
& =\alpha_{31}^{103} \frac{1}{2}\left(4 L_{3}-1\right)\left(4 L_{3}-2\right) \boldsymbol{w}_{31}, \\
\boldsymbol{W}_{11}=\boldsymbol{W}_{31}^{103} & =\alpha_{31}^{103} S_{1}^{4}\left(L_{1}\right) P_{0}^{4}\left(L_{2}\right) S_{3}^{4}\left(L_{3}\right) \boldsymbol{w}_{31} \\
\boldsymbol{W}_{12}=\boldsymbol{W}_{31}^{202} & =\alpha_{31}^{202} S_{2}^{4}\left(L_{1}\right) P_{0}^{4}\left(L_{2}\right) S_{2}^{4}\left(L_{3}\right) \boldsymbol{w}_{31} \\
& =\alpha_{31}^{202}\left(4 L_{1}-1\right)\left(4 L_{3}-1\right) \boldsymbol{w}_{31}, \\
\boldsymbol{W}_{13}=\boldsymbol{W}_{31}^{301} & =\alpha_{31}^{301} S_{3}^{4}\left(L_{1}\right) P_{0}^{4}\left(L_{2}\right) S_{1}^{4}\left(L_{3}\right) \boldsymbol{w}_{31} \\
& =\alpha_{31}^{301} \frac{1}{2}\left(4 L_{1}-1\right)\left(4 L_{1}-2\right) \boldsymbol{w}_{31}, \\
\boldsymbol{W}_{15}=\boldsymbol{W}_{31}^{211} & =\alpha_{31}^{211} S_{2}^{4}\left(L_{1}\right) P_{1}^{4}\left(L_{2}\right) S_{1}^{4}\left(L_{3}\right) \boldsymbol{w}_{31}\left(4 L_{2}\right)\left(4 L_{1}-1\right) \boldsymbol{w}_{31} . \\
\boldsymbol{W}_{14}=\boldsymbol{W}_{31}^{112} & =\alpha_{31}^{112} S_{1}^{4}\left(L_{1}\right) P_{1}^{4}\left(L_{2}\right) S_{2}^{4}\left(L_{3}\right) \boldsymbol{w}_{31} \\
& =\alpha_{31}^{112}\left(4 L_{2}\right)\left(4 L_{3}-1\right) \boldsymbol{w}_{31}, \\
\boldsymbol{H}_{11} &
\end{aligned}
$$

The vector shape functions in $3 \mathrm{D}$ can be constructed as the extension of the above presented 2D realization. Three-dimensional zeroth order edge shape functions can be constructed as,

$$
\begin{aligned}
& \boldsymbol{W}_{1}=l_{1}\left(N_{1} \nabla N_{2}-N_{2} \nabla N_{1}\right) \delta_{1}, \\
& \boldsymbol{W}_{2}=l_{2}\left(N_{2} \nabla N_{3}-N_{3} \nabla N_{2}\right) \delta_{2}, \\
& \boldsymbol{W}_{3}=l_{3}\left(N_{3} \nabla N_{1}-N_{1} \nabla N_{3}\right) \delta_{3}, \\
& \boldsymbol{W}_{4}=l_{4}\left(N_{1} \nabla N_{4}-N_{4} \nabla N_{1}\right) \delta_{4}, \\
& \boldsymbol{W}_{5}=l_{5}\left(N_{2} \nabla N_{4}-N_{4} \nabla N_{2}\right) \delta_{5}, \\
& \boldsymbol{W}_{6}=l_{6}\left(N_{3} \nabla N_{4}-N_{4} \nabla N_{3}\right) \delta_{6} .
\end{aligned}
$$

Here $l_{i}$ (Fig. 26) is the length of the edges and it is used to normalize the edge shape function according to (104). The value of $\delta_{i}$ is also equal to \pm 1 depending on whether the local direction of the edge is the same as the global direction or opposite. The edge definition employed in my analysis can be seen in Fig. 26. 
If the approximation of the vector function $\boldsymbol{T}$ is known along the edges of the mesh, then (98) can be used to interpolate the function anywhere and in linear case $k=6$.

To construct higher order vector basis functions, the points of interpolation polynomials are arranged in a pyramid format to build an applicable numbering scheme $(I, J, K, L)$ and $I, J, K, L=0,1, \cdots, n+2$, where $n$ is the order of the element. The illustration of numbering scheme in $3 \mathrm{D}$ is not easy, but it can be construct as follows. Let us imagine the same numbering scheme on the triangular facets of the tetrahedron as in Fig. 31-Fig. 33 and the integers $I, J, K$ and $L$ can be set up according to the facets.

The vector shape functions of order $n$ are given as,

$$
\begin{aligned}
& \boldsymbol{W}_{12}^{I J K L}=\alpha_{12}^{I J K L} S_{I}^{n+2}\left(L_{1}\right) S_{J}^{n+2}\left(L_{2}\right) P_{K}^{n+2}\left(L_{3}\right) P_{L}^{n+2}\left(L_{4}\right) \boldsymbol{w}_{12}, \\
& \boldsymbol{W}_{23}^{I J K L}=\alpha_{23}^{I J K L} P_{I}^{n+2}\left(L_{1}\right) S_{J}^{n+2}\left(L_{2}\right) S_{K}^{n+2}\left(L_{3}\right) P_{L}^{n+2}\left(L_{4}\right) \boldsymbol{w}_{23}, \\
& \boldsymbol{W}_{31}^{I J K L}=\alpha_{31}^{I J K L} S_{I}^{n+2}\left(L_{1}\right) P_{J}^{n+2}\left(L_{2}\right) S_{K}^{n+2}\left(L_{3}\right) P_{L}^{n+2}\left(L_{4}\right) \boldsymbol{w}_{31}, \\
& \boldsymbol{W}_{14}^{I J K L}=\alpha_{14}^{I J K L} S_{I}^{n+2}\left(L_{1}\right) P_{J}^{n+2}\left(L_{2}\right) P_{K}^{n+2}\left(L_{3}\right) S_{L}^{n+2}\left(L_{4}\right) \boldsymbol{w}_{14}, \\
& \boldsymbol{W}_{24}^{I J K L}=\alpha_{24}^{I J K L} P_{I}^{n+2}\left(L_{1}\right) S_{J}^{n+2}\left(L_{2}\right) P_{K}^{n+2}\left(L_{3}\right) S_{L}^{n+2}\left(L_{4}\right) \boldsymbol{w}_{24}, \\
& \boldsymbol{W}_{34}^{I J K L}=\alpha_{34}^{I J K L} P_{I}^{n+2}\left(L_{1}\right) P_{J}^{n+2}\left(L_{2}\right) S_{K}^{n+2}\left(L_{3}\right) S_{L}^{n+2}\left(L_{4}\right) \boldsymbol{w}_{34} .
\end{aligned}
$$

The parameters denoted by $\alpha$ are normalization factors, which must have the value such that the line integral of the vector shape function $\boldsymbol{W}_{a b}^{I J K L}$ is equal to 1 on the edge pointing from node $a$ to node $b$.

The number of edge shape functions when defining the $n^{\text {th }}$ order family is

$$
k=\frac{(n+1)(n+3)(n+4)}{2} .
$$

For each interpolation point on the edge, there is one corresponding vector shape function, which means $6(n+1)$ functions. For each interpolation point on the face of a tetrahedron there are three vector functions, but one of them is depending on the other two and it must be discarded, finally there are $4 n(n+1)$ vector shape functions defined on the four facets. For each interpolation points inside the element there are six basis functions. A $3 \mathrm{D}$ vector has only three degree of freedom, that is why three vector basis functions must be discarded resulting in $n(n-1)(n+1) / 2$ vector basis functions. Totally, there are $6(n+1)+4 n(n+1)+n(n-1)(n+1) / 2=(n+1)(n+3)(n+4) / 2$ vector shape functions. There are $k=6, k=20$ and $k=45$ shape functions for $n=0, n=1$ and $n=2$, respectively.

As an example, the following vector shape functions can be set up when $n=1$, $\boldsymbol{W}_{12}^{2100}, \boldsymbol{W}_{12}^{1200}, \boldsymbol{W}_{23}^{0210}, \boldsymbol{W}_{23}^{0120}, \boldsymbol{W}_{31}^{1020}, \boldsymbol{W}_{31}^{2010}, \boldsymbol{W}_{14}^{2001}, \boldsymbol{W}_{14}^{1002}, \boldsymbol{W}_{24}^{0201}, \boldsymbol{W}_{24}^{0102}$, $\boldsymbol{W}_{34}^{0021}, \boldsymbol{W}_{34}^{0012}, \boldsymbol{W}_{12}^{1110}, \boldsymbol{W}_{31}^{1110}, \boldsymbol{W}_{14}^{1011}, \boldsymbol{W}_{34}^{1011}, \boldsymbol{W}_{23}^{0111}, \boldsymbol{W}_{24}^{0111}, \boldsymbol{W}_{12}^{1101}, \boldsymbol{W}_{24}^{1101}$. 
M. Kuczmann - Acta Technica Jaurinensis, Vol.8., No.4., pp. 347-383, 2015

\section{References}

[1] Kuczmann M, Iványi A: The Finite Element Method in Magnetics, Academic Press, Budapest, 2008. 